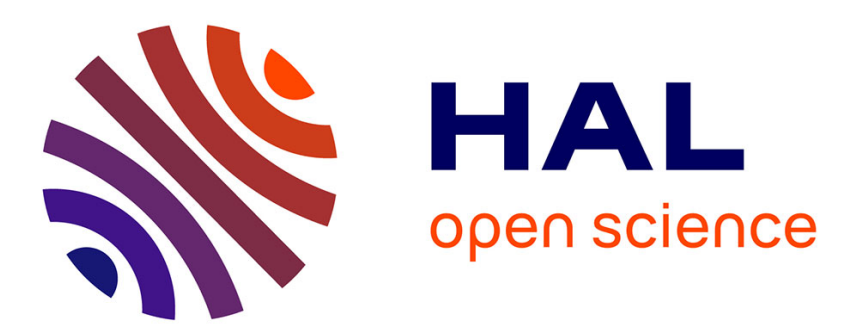

\title{
Les parfumeries en Campanie antique
}

Nicolas Monteix, Jean-Pierre Brun

\section{To cite this version:}

Nicolas Monteix, Jean-Pierre Brun. Les parfumeries en Campanie antique. Jean-Pierre Brun. Artisanats antiques d'Italie et de Gaule. Mélanges offerts à Maria Francesca Buonaiuto, Centre Jean Bérard, pp.115-133, 2009, Collection du Centre Jean Bérard, 32 ; Archéologie de l'artisanat antique, 2. halshs-00619020

\section{HAL Id: halshs-00619020 https://shs.hal.science/halshs-00619020}

Submitted on 5 Sep 2011

HAL is a multi-disciplinary open access archive for the deposit and dissemination of scientific research documents, whether they are published or not. The documents may come from teaching and research institutions in France or abroad, or from public or private research centers.
L'archive ouverte pluridisciplinaire HAL, est destinée au dépôt et à la diffusion de documents scientifiques de niveau recherche, publiés ou non, émanant des établissements d'enseignement et de recherche français ou étrangers, des laboratoires publics ou privés. 


\title{
Les parfumeries en Campanie antique
}

\author{
Jean-Pierre Brun* et Nicolas Monteix**
}

\author{
À la fille du parfumeur de la Piazza Trieste e Trento
}

"Cette terre ne cesse jamais d'enfanter, ce qui fait dire communément que la Campanie produit plus de parfum que les autres pays ne produisent d'huile» écrit Pline au livre XVIII, 111 de son Histoire Naturelle. La nature et les hommes avaient doté la Campanie de tous les dons nécessaires à la fabrication des parfums : une huile d'olive exceptionnelle, des fleurs, des liens commerciaux avec l'Orient et un savoir-faire également d'origine orientale. L'huile de Vénafre, au nord de la Campanie, était particulièrement recherchée en parfumerie pour son aptitude à recevoir les fragrances (Pline, $N$. H. XV, 8). Le connaisseur décelait, en arrièreplan des senteurs aromatiques, l'odeur subtile de l'huile de Vénafre qui lui servait de base (Martial, XIII, 101). Des innombrables ingrédients entrant dans la composition des parfums, la Campanie cultivait les plus couramment utilisés, les fleurs, et importait les autres, résines et écorces tropicales, grâce à ses nombreux ports en relations constantes avec l'Orient. Parmi les fleurs, les roses tenaient un rôle prépondérant que Pline souligne à plusieurs reprises en rappelant avec bien d'autres auteurs qu'elles sont très largement cultivées ( $N$. $H$. III, 40 ; XIII, 26 ; XVIII, 111 ; XXI,16). Toutes ces potentialités seraient restées inexploitées sans un savoirfaire attesté dès la période républicaine dans certaines villes comme Capoue (Frederiksen 1959, p. 110-111). Durant l'Empire, elle maintiendra sa réputation au point que les fabricants d'onguents (unguentarii) seront appelés seplasiarii du nom d'une des places de Capoue où ils produisaient et vendaient des huiles parfumées.
Nul doute que les relations constantes de la Campanie, notamment de Pouzzoles, avec l'Orient et en particulier l'Égypte n'aient été le moteur du développement de l'industrie des parfums (Camodeca 1979). D’Orient venaient les aromates, les recettes et les hommes instruits dans cet art. Dans le roman de Pétrone, Trimalcion dit avoir fait le commerce des parfums (Sat. 76) dans un discours où les lieux communs de l'enrichissement sont le commerce du vin, des parfums et des esclaves.

À l'époque tardo-républicaine et sous l'Empire, l'emploi des huiles parfumées était très large. Tout d'abord, le parfum remplissait sa fonction de vecteur de la séduction. Pline dit justement Summa commendatio eorum ut, transuente femina, odor invitet etiam alius agentis ( le plus haut titre de recommandation d'un parfum est, sur le passage d'une femme qui le porte, d'attirer par ses effluves même ceux qui sont occupés à tout autre chose " N. H. XIII, 20).

Plus généralement et depuis l'époque grecque archaïque au moins, les huiles parfumées étaient

\footnotetext{
* Centre Jean Bérard, USR 3133 (CNRS-École française de Rome). Pendant ces dix dernières années, Maria Francesca a été une collaboratrice très proche et, bien plus que cela, une amie sur qui compter en toutes circonstances [JPB].

** École française de Rome. Depuis bientôt dix ans que je viens travailler à Herculanum et Pompéi, il m'apparaît évident qu'aucune de mes recherches n'aurait été possible sans la gentillesse et la disponibilité de Maria Francesca. Puissent mes quelques remarques sur Herculanum atténuer une dette qui n'a cessé de croître d’année en année [NM].
} 


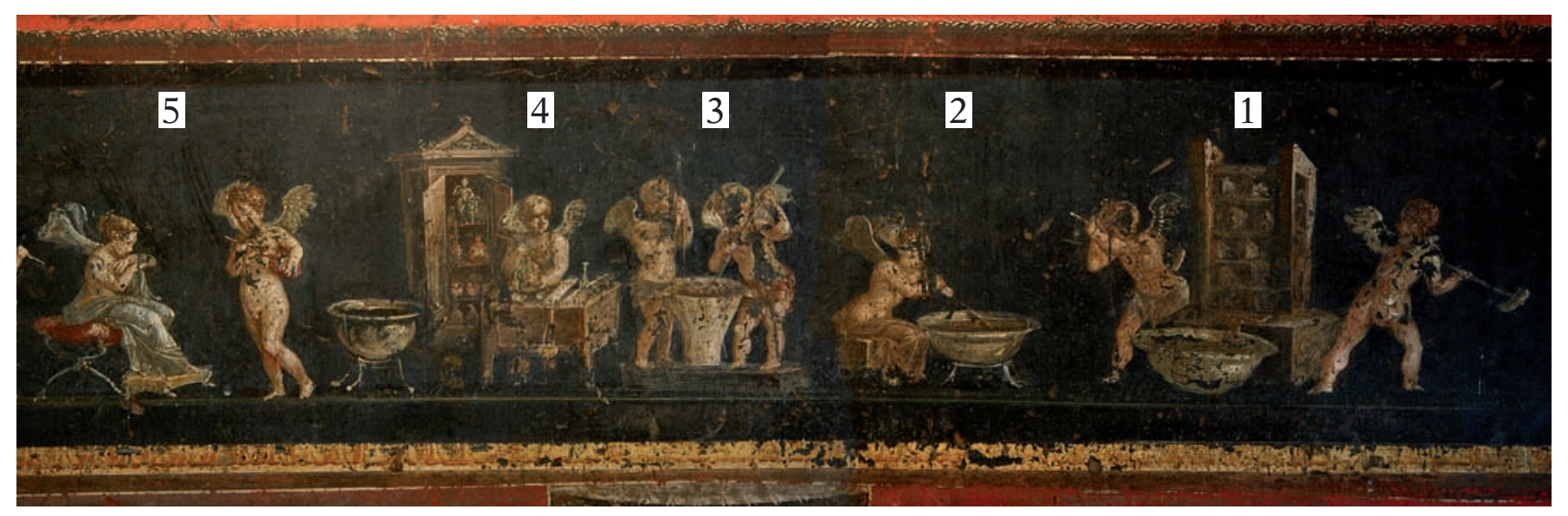

Fig. 1. La préparation des parfums dans une boutique. Peinture de l'oecus de la Maison des Vettii. De droite à gauche : 1. extraction de l'huile à parfum avec une presse à coins, 2. enfleurage à chaud de l'huile dans un chaudron sur un foyer, 3. broyage des ingrédients, notamment des résines, dans un mortier, 4. comptoir de vente avec balance, papyrus et, dans l'armoire à l'arrière-plan, files de flacons à parfums et statuette de Vénus, 5. un vendeur fait essayer un parfum à une cliente sur son poignet (cliché J.-P. Brun)

utilisées pour les soins du corps. Les athlètes s'enduisaient d'huile et après l'exercice se faisaient masser souvent avec des huiles parfumées. Il en allait de même pendant le bain et surtout après le bain. On ne concevait pas un séjour aux thermes sans qu'il soit suivi d'une onction de parfum; on apportait souvent un flacon avec soi pour cet usage.

Le plaisir, l'hygiène et la thérapeutique se combinaient car les huiles parfumées constituaient l'une des bases de la pharmacopée, comme le mentionne le Digeste : Unguentum legatis, non tantum ea legata videntur, quibus ungimur voluptatis causa, sed valetudinis (XXXIV, 2, 21, 1) et valetudinis causa (XXXIV, 2, 3, 12). Cette activité proprement pharmaceutique est une branche inséparable et fondamentale de la parfumerie antique. Pline explique que les médecins incapables de préparer euxmêmes leurs remèdes et de surveiller les seplasiarii (au sens de parfumeurs fabricants de drogues), laissent à ceux-ci la possibilité d'écouler des marchandises frelatées (XXXIV, 108). Dioscoride, dans son ouvrage médical, notamment au livre I, donne une longue liste de recettes d'huiles parfumées avec leurs indications thérapeutiques.

Lorsque, malgré les efforts des médecins et les drogues des pharmaciens, la mort avait fait son œuvre, c'est aussi vers les parfums qu'on se tournait afin de préparer le cadavre pour les funérailles. Citons Perse «notre jeune homme posé sur un lit de parade et tout enduit de parfum, est étendu à la porte, les pieds devant» (Satires, III, 104 sq.) et Apulée : "Asclépiade contemplait ce malheureux dont les membres étaient déjà saupoudrés d'aromates, dont le visage était déjà recouvert d'une pommade odorante et en l'honneur de qui on préparait déjà le repas funèbre» (Florides, IV, 19). L'urne funéraire était parfumée avant de recevoir les cendres du défunt (Perse, Satires, VI, 34 sq.) et, lors des funérailles de notables, il était courant de brûler des quantités notables d'encens et de répandre des parfums. Lors de celles d'Obellius Firmus à Pompéi 30 livres d'encens furent brûlés et 1000 sesterces de parfum dépensés (De Caro 1979, p. 61). On enduisait aussi la stèle funéraire ellemême comme d'ailleurs stèles et statues de culte comme partout ailleurs en Méditerranée, notamment en Grèce.

En sus de ces usages corporels ou religieux, le parfum était employé pour parfumer le linge (Martial, VIII, 3, 10 ; Clément d'Alexandrie, Paed. II, 8), les banquettes des thermes (Pline, XIII, 22), les participants des banquets (Horace, Odes, II, 7, 23) et les spectateurs des théâtres (Sénèque, Ep. 90 ; Martial, V, 25 ; Spect. III, 8)

La Campanie se distinguait, de par ses origines grecque et étrusque, ses liens commerciaux avec l'Orient et ses productions propres, par un usage intense des parfums. Les parfums et les parfumeries sont donc des thèmes qui auraient dû y susciter de nombreuses recherches, or elles se sont limitées, 


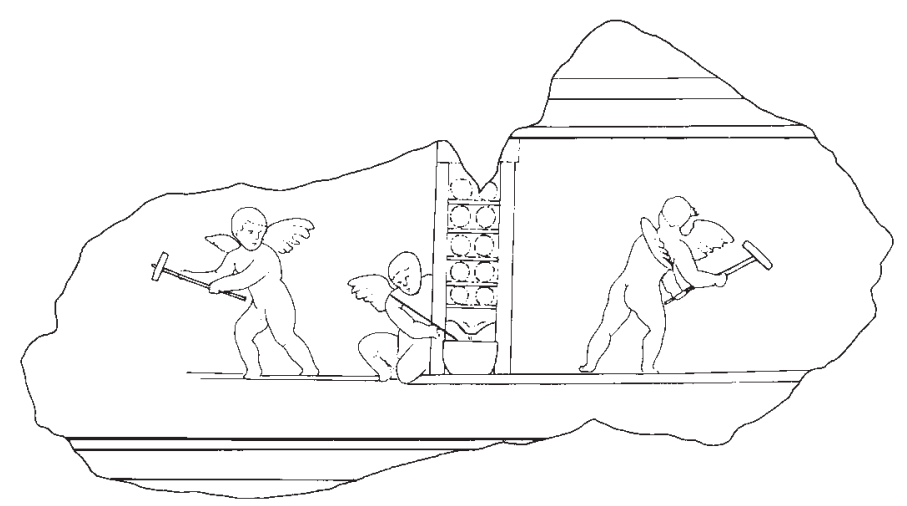

Fig. 2. Pompéi. Peinture conservée au Fitzwilliam Museum de Cambridge montrant des amours frappant avec des maillets sur une presse à coins (d'après Mattingly 1990)

faute de clefs interprétatives, à l'étude des inscriptions ${ }^{1}$ et à celle des flacons ${ }^{2}$. Jusqu'à la fin des années 1990, peu de travaux ont porté sur les parfumeries elles mêmes mis à part ceux de W. Jashemsky, puis de David Mattingly sur Pompéi. W. Jashemsky s'était intéressée aux cultures florales et leurs débouchées, décoration et parfumerie. D. Mattingly s'est surtout penché sur l'iconographie des parfumeries, étudiant quatre peintures de Pompéi (Casa dei Vettii, ici fig. 1, maison VII, 7, 5, et peinture conservée au Fitzwilliam Museum de Cambridge, ici fig. 2) et d'Herculanum (Casa dei Cervi, ici fig. 3) ${ }^{3}$. Depuis, les recherches se sont multipliées sur des installations anciennement dégagées mais mal comprises, à Herculanum, à Pompéi et à Paestum. Elles offrent une vision renouvelée des ateliers de parfumeurs qui devaient certainement vendre directement leurs productions.

\section{Herculanum}

En juin 1928, une presse à vis en bois a été mise au jour dans la boutique III, 10, située à l'angle nordest de la Casa del Tramezzo di legno à Herculanum. Jusqu'à présent, elle a été interprétée dans le cadre de l'artisanat du textile : elle aurait servi à repasser les étoffes à la fin du processus de nettoyage. Cette interprétation n'a guère été justifiée ou approfondie, en dépit d'une première description comme presse à huile $^{4}$. Lors de la publication définitive de ses fouilles, A. Maiuri reprend cette idée, en ajoutant simplement que la presse est l'unique instrument de

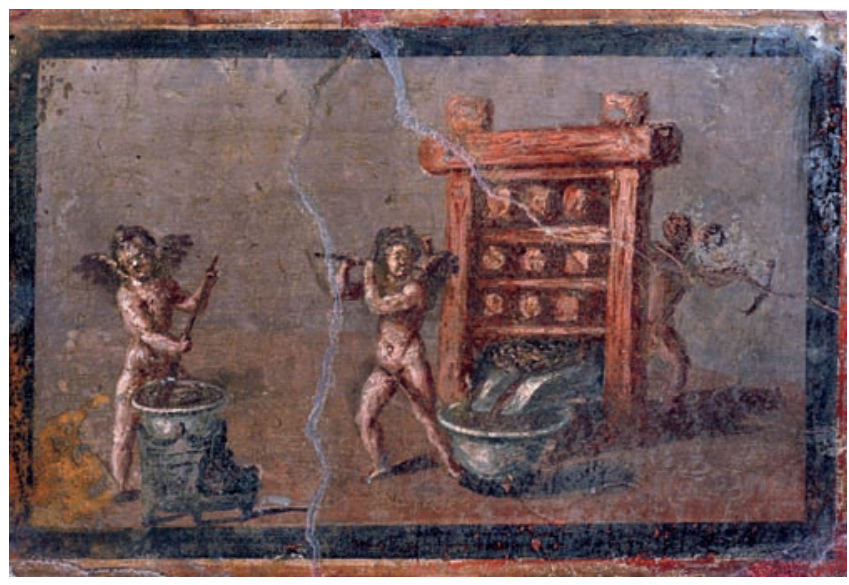

Fig. 3. Herculanum, Casa dei Cervi, peinture montrant la fabrication de l'huile avec une presse à coins et l'enfleurage sur un fourneau (Musée archéologique national de Naples; cliché J.-P. Brun)

travail du supposé lanarius officiant dans ce local. Aucune argumentation n'est développée, l'auteur se contentant de renvoyer à d'ultérieures explications, jamais données ${ }^{5}$. Quelques fragiles éléments de comparaison sont proposés dans la note qui conclut ce paragraphe : A. Maiuri renvoie aux ouvrages de J. Overbeck et de V. Spinazzola pour suggérer une similitude entre ce pressoir et celui représenté sur une peinture mise au jour dans la fullonica de L. Veranius Hypsaeus (VI 8, 2.20-21) à Pompéi (Maiuri 1958, p. 471, note 33). À partir d'une des-

1 Par exemple ceux de Pouzzoles, évoqués dans l'article de Camodeca 1979, p. 24-25.

2 Voir par exemple l'étude des contenus de bouteilles et flacons publiée par Scatozza Höricht et alii 1993.

3 À ces quatre peintures, il convient d'ajouter celle de la Villa Imperiale qui montre une scène d'enfleurage : un amour brasse le parfum dans un chaudron sur un foyer : Pappalardo 1987, p. 130.

4 Concernant la première interprétation comme presse à huile, elle est clairement indiquée dans un article du Times, en date du 7 décembre 1928 (p. 15) : «Interesting also is a wooden oil press used for crushing olives and of a type identical with those used to this day ». Cette information paraît correspondre à un télégramme de presse, relayé par plusieurs autres journaux. Ce n'est que dans un deuxième temps que cette interprétation est modifiée (Maiuri 1931), avant de devenir le seul discours la concernant (Maiuri 1932 : 60 et fig. p. 58 ; Maiuri 1936, p. 34).

5 « [...] Il suo carattere e la sua destinazione ci sono fortunatamente attestati [...] da un torchio di legno che, come diremmo in seguito [nous soulignons], per la sua forma e le sue dimensioni, non può essere altro che un pressorium per i panni [...] " (Maiuri 1958, p. 220). 


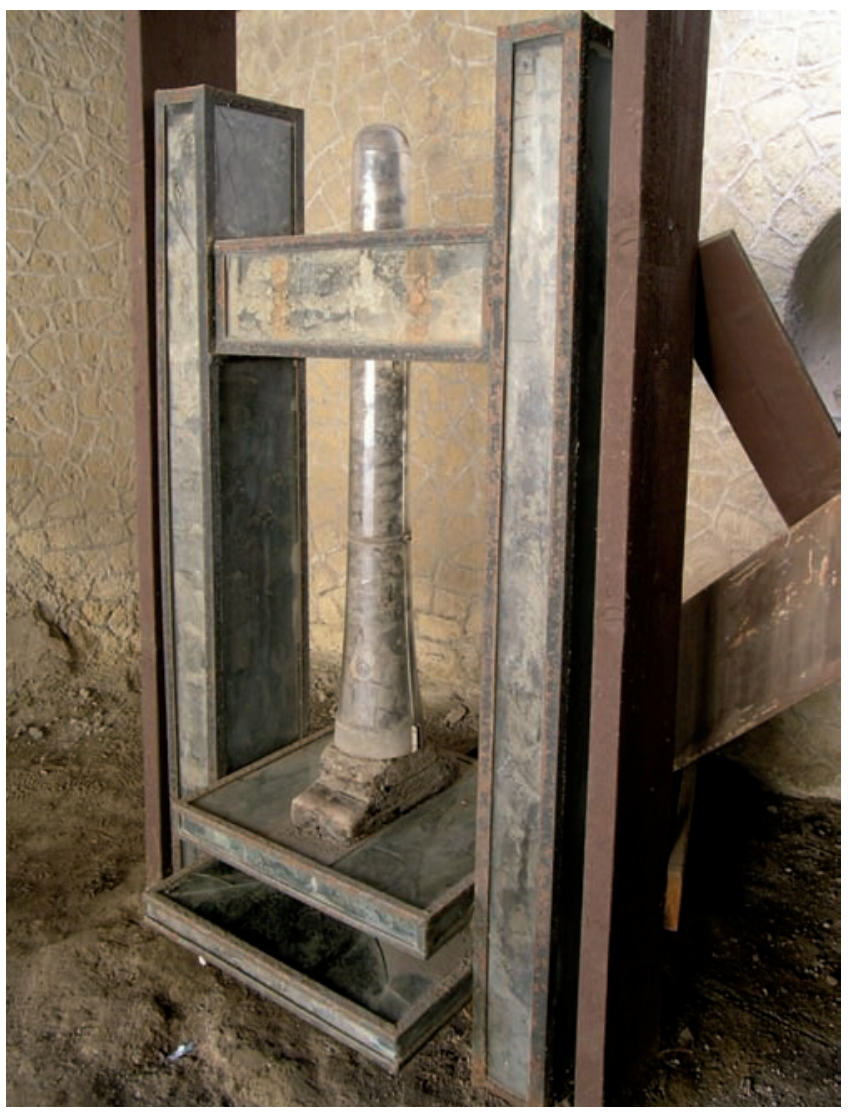

Fig. 4. Presse à vis découverte dans la boutique III, 10 à Herculanum (cliché N. Monteix)

cription de la presse d'Herculanum, utilisant conjointement les vestiges en place et les journaux de fouille (GSE), il est possible de revoir cette interprétation.

La presse découverte en III, 10 est un cadre en bois formé par deux montants de $12 \mathrm{~cm}$ de large

${ }^{6}$ Selon les journaux de fouille, cette hauteur est minimale : sans qu'aucune explication ne soit fournie, les deux piliers ne touchent pas le sol. GSE 1928 : « 20 luglio. [...] Il congegno di legno carbonizzato è stato isolato e risulta una pressa. I due pilastri esterni sono attualmente alti m. 1.50 ma erano ancora più alti, perché la parte inferiore ora non tocca il pavimento ".

7 Lors de la description finale de la Casa del Tramezzo di legno, effectuée après la restauration, les données métriques de la presse ont subi quelques modifications. Les piliers font désormais $171 \mathrm{~cm}$ de haut, leur écartement est porté de $84 \mathrm{~cm}$ à $90 \mathrm{~cm}$. Ces transformations sont certainement à mettre en relation avec les "véritables cages vitrées » (Maiuri 1932, p. 60) utilisées pour protéger la presse.

8 Hauteur : $21 \mathrm{~cm}$; épaisseur : $23 \mathrm{~cm}$. La description faite dans les GSE n'est pas en adéquation avec la reconstitution visible actuellement sur le site : GSE 1928 : « 20 luglio. [...] A m. 0.26 dall'estremità superiore si trova un incasso nel quale è fissato un traversone che è lungo m. 1.05 pari alla distanza che

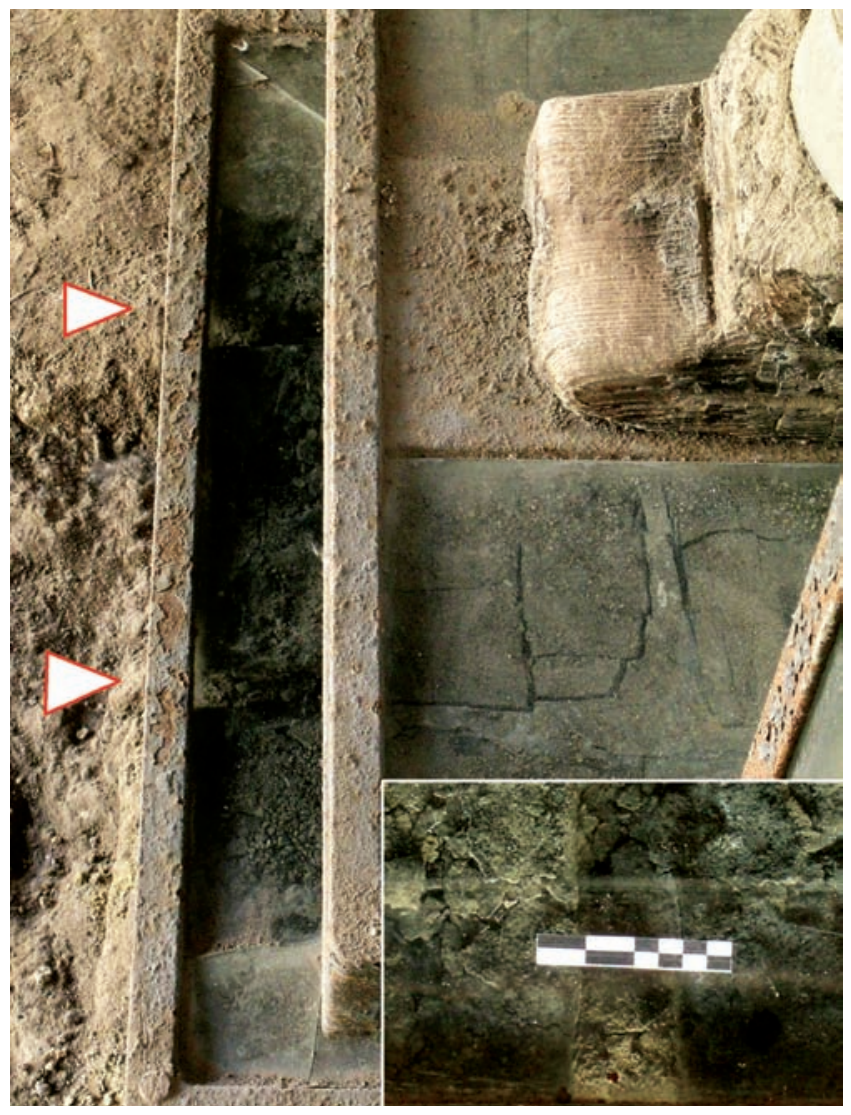

Fig. 5. Vue du plateau et de la maie de la presse (Les flèches indiquent les rainures excavées sur la surface supérieure de la maie. En encadré, vue zénithale d'une des rainures.)

pour $27 \mathrm{~cm}$ d'épaisseur (fig. 4 et 5). Leur hauteur conservée est de $150 \mathrm{~cm}^{6}$. Aucune précision n'est donnée quant à leur mode de fixation dans le $\mathrm{sol}^{7}$. Ces jumelles sont reliées par un banc horizontal long de $105 \mathrm{~cm}^{8}$. L'assemblage précis de ces diffé-

passa dai due estremi dei pilastri. [...] Al centro di esso e cioè a m. 0.26 dalla faccia interna di ciascun pilastro vi è piazzato un cilindro superficialmente circondato da una spirale [...] ». Tel qu'il est décrit, le banc ne devrait pas dépasser des jumelles dont l'écartement, mesuré à l'extérieur, serait justement de $105 \mathrm{~cm}$. Pourtant, avec les indications qui sont données quant à la localisation de l'écrou un rapide calcul amène à réduire cet écartement des jumelles: 2 x $26 \mathrm{~cm}$ (distance intérieur des jumelles - écrou) $+6 \mathrm{~cm}$ diamètre de la vis $+2 \times 12 \mathrm{~cm}$ (épaisseur des jumelles) = $82 \mathrm{~cm}$. Il s'ensuit que soit le banc a été coupé au remontage - il devrait dépasser d'environ $10 \mathrm{~cm}$ de chaque côté des jumelles soit l'indication de la mesure entre l'écrou et les jumelles est erronée. En corrigeant cette dernière - de 26 à $36 \mathrm{~cm}$ - et en rectifiant le diamètre de la vis à $9 \mathrm{~cm}$ - tel que mesuré sur place - les dimensions sont de nouveau en accord avec la première mention d'écartement des jumelles. Il nous semble peu probable que cette erreur soit liée à un problème d'écriture concernant la distance entre ces dernières. 
rentes pièces entre elles est difficile à établir à cause des restaurations. Toutefois, la description des GSE indique clairement qu'une mortaise a été ménagée dans chaque jumelle. Le maintien du banc aux jumelles paraît avoir été assuré par l'enfoncement de coins ${ }^{9}$. Au centre de cette pièce horizontale, un écrou permettant le passage d'une vis de $9 \mathrm{~cm}$ de diamètre a été creusé. Bien que les journaux de fouille décrivent un banc monoxyle, il est en fait constitué par deux traverses assemblées grâce à deux bardes en métal disposées de part et d'autre de l'écrou ${ }^{10}$. La vis est haute de $93 \mathrm{~cm}$, mais son amplitude maximale est limitée à environ $60 \mathrm{~cm}$, à cause de l'augmentation de son diamètre à sa base. Le plateau de transmission de la pression est une planche carrée de $62 \mathrm{~cm}$ de côté, épaisse de $7 \mathrm{~cm}$. La maie est monoxyle et épaisse de $10 \mathrm{~cm}$. Deux rainures longitudinales de section quadrangulaire ont été pratiquées sur sa face supérieure. Comme le font très justement remarquer les rédacteurs des GSE, ces deux saignées permettent l'écoulement d'un liquide ${ }^{11}$. Aucun élément n'aide à définir le mode de fixation de la maie aux jumelles, faute de description ou de mise au jour d'une pièce de bois transversale permettant de fermer le cadre dans lequel est inséré la vis. L'ensemble paraît avoir été fixé par rapport aux murs par le biais de plusieurs poutrelles d'un diamètre de $6 \mathrm{~cm}^{12}$. Enfin, bien qu'obstrués, les deux orifices orthogonaux servant de logement à la barre de manœuvre sont encore visibles. Ils se situent dans la partie élargie de la vis. Pour complé-

${ }^{9}$ GSE 1928 : « 20 luglio. [...] All'estremità esterna superiore di ciascun pilastro eravi un travicello, quello al lato sinistro era a guisa di maniglia e l'altro era inclinato nell'interno, in maniera che in alto formavano un angolo acuto [...] ». Cette description reste passablement incompréhensible. L’observation détaillée des vestiges en place n'a pas permis de la clarifier.

10 Ces deux bandes métalliques, passablement corrodées sont encore visibles sur la presse. L'usage de deux traverses assemblées permet de faciliter le creusement du pas de vis.

11 GSE 1928 : « 24 luglio. [...] Il pezzo superiore che serviva come pressa ed era attaccato alla vite, mediante un perno di ferro non fisso era lungo m. 0.62, e lo spessore m. 0.07, il secondo pezzo di legno che formava la base della pressa aveva la medesima lunghezza del primo ma lo spessore era di m. 0.10. Sulla faccia superiore di quest'ultimo pezzo si notano dei piccoli canaletti larghi m. 0.02, essi servivano forse per far colare il liquido $[\ldots] »$.

12 GSE 1928 : « 20 luglio. [...] Per mantenere fissa la pressa vi erano dei travicelli del diametro di m. 0.06 che erano a mo' di puntello alle fauci laterali [...] ». Les importants travaux de restauration des murs qui se sont développés dans cette boutique empêchent de cerner le point de fixation de ces poutrelles. De la même façon, le coffrage en bois et en verre entourant la presse ter la description de ce dispositif, il nous faut mentionner la fosse excavée sous le pressoir ${ }^{13}$. Ses dimensions ne sont jamais indiquées dans les GSE. Elle contenait une matière noire non identifiée lors de sa mise au jour ${ }^{14}$. En l'état actuel, il est impossible d'observer cette cuve : après sa consolidation, la presse a été repositionnée très exactement à l'endroit où elle a été découverte. Pour résumer, les caractéristiques de la structure de pressage mise au jour dans la boutique III 10 sont donc les suivantes : il s'agit d'une presse à une seule vis centrale, dont la maie carrée en bois est pourvue de canaux permettant l'écoulement d'un liquide. Concernant son mode de fixation au sol, seul l'enfoncement des montants dans le sol paraît l'avoir assuré. Il conviendrait donc de restituer une seconde traverse pour soutenir la maie.

Faisons un rapide excursus sur les presses utilisées dans les fouleries. À partir des données issues de la fullonica de Stephanus (I 6, 7) à Pompéi, où des éléments d'une presse ont été mis au jour en juillet 1912 (NSc 1912, p. 248-249), complétée par la représentation provenant de la fullonica de L. Veranius Hypsaeus (VI 8, 2.20-21; ici fig. 6) ${ }^{15}$ et avec certains passages du traité de mécanique d'Héron d'Alexandrie ${ }^{16}$, il est possible de proposer une restitution de presse à vêtements (fig. 7). Sans développer les détails de cette analyse, celle-ci nous permet de dresser une comparaison avec la presse d'Herculanum d'un point de vue technique ${ }^{17}$. Le cadre est formé par l'enchâssement d'un banc

ne permet pas de confirmer - ou d'infirmer - le lien que ces dernières pouvaient entretenir avec celle-ci.

13 GSE 1928 : « 20 luglio. [...] A quanto pare sotto la pressa si trova una vaschetta in muratura, le dimensioni non sono ancora conosciute perché non del tutto sterrata [...] ".

14 GSE 1928 : " 24 luglio. [...] Nelle cunetta è stato raccolto materia nera che deve essere esaminata [...] »; GSE 1929 : " 27 maggio. [...] Nella cunetta fu raccolto materia nera [...] ».

15 Cette fresque est désormais conservée au Museo Archeologico Nazionale di Napoli (MANN, Inv. n9774). La bibliographie la concernant et une analyse stylistique ont été récemment proposées par Th. Fröhlich (1991, p. 229-236).

16 Héron, Mec., 3, 19-21 (cf. Carra de Vaux 1988). Ces paragraphes décrivent une presse à huile à deux vis. Ce sont essentiellement les parties concernant le creusement de deux écrous dans un banc monoxyle qui ont été utilisés dans le cadre de cette restitution.

17 Une première restitution de la presse mise au jour dans la fullonica de Stephanus, qui se fonde également sur le parallèle entre les vestiges découverts dans cet atelier et la fresque de L. Veranius Hypsaeus, a été proposée par V. Spinazzola (1953, p. 777, fig. 764-766). Une analyse détaillée de celle-ci montre qu'elle est techniquement non fonctionnelle : l'utilisation des vis de cette presse entraînerait sa dislocation immédiate. 


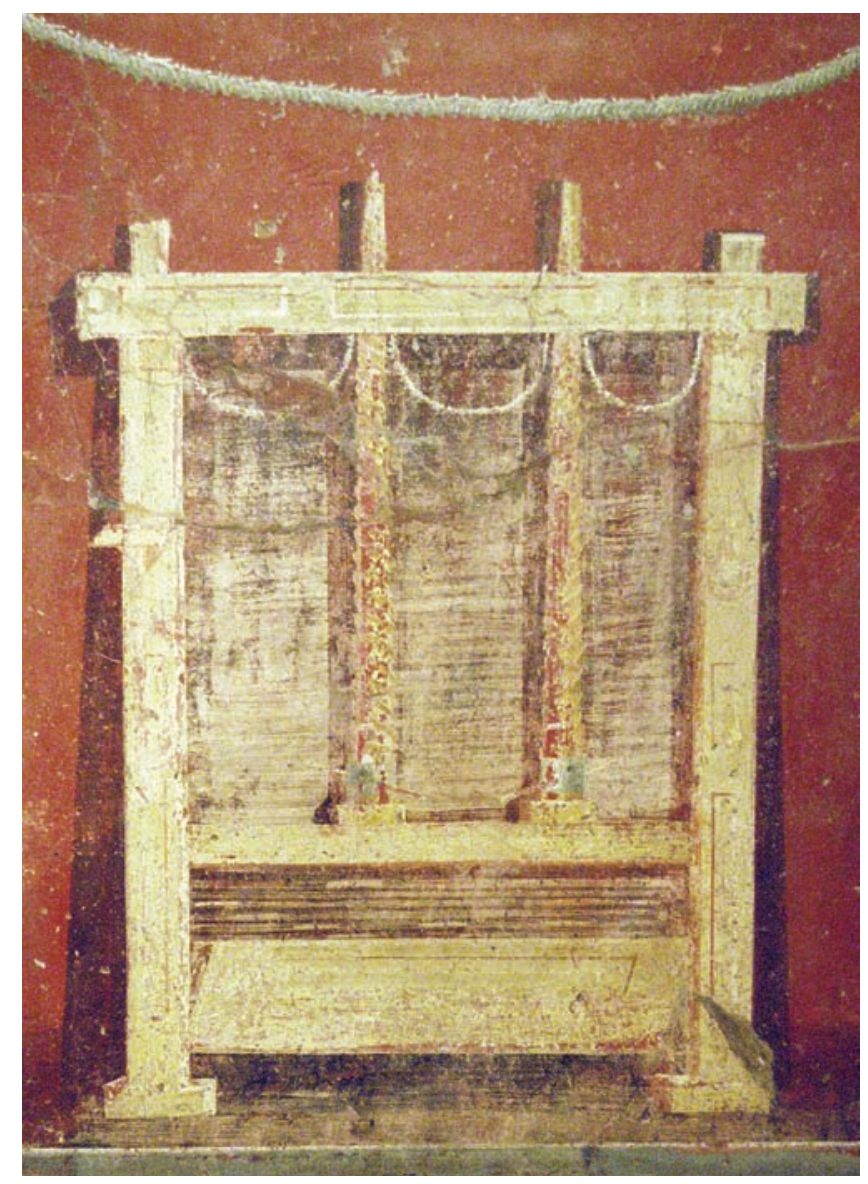

Fig. 6. Fresque recouvrant le pilier nord à l'est du péristyle de la fullonica de L. Veranius Hypsaeus (Pompéi, VI 8, 2.20-21), presse à vêtements (MANN, Inv. 9774, cliché N. Monteix)

monoxyle dans deux jumelles profondément enfoncées dans deux fosses dont les dimensions ont été notées lors de la fouille ${ }^{18}$. La traverse inférieure, qui devait s'insérer dans des mortaises pratiquées dans les jumelles, est en contact avec le sol. Celui-ci a été excavé sur une profondeur de $2 \mathrm{~cm}$ pour assurer une meilleure assise à cette pièce ${ }^{19}$. La base des deux vis est enserrée dans une lanterne constituée de deux frettes métalliques reliées par des plaques percées de trous. Pour permettre l'abaissement du plateau, les vis devaient être actionnées simultanément. Au-delà des différences non négligeables qui distinguent cette presse de celle d'Herculanum dimension, nombre de vis, assemblage du cadre -, la principale d'entre elles reste l'absence de toute fosse permettant de recueillir un liquide (fig. 7). En dépit des difficultés de restitution de la chaîne opératoire de la foulerie en utilisant les textes antiques, rien ne permet de supposer les tissus gorgés d'eau lors de leur passage sous la presse : si l'on suit les indications de Martial, le but de l'opération n'est autre que de lustrer les pièces d'étoffe, certainement pas de les essorer ${ }^{20}$. De plus, aucun canal d'écoulement n'est visible sur la fresque et aucune fosse ou rigole de collecte n'a été mise en évidence autour des jumelles exhumées en 1912, autant de signes corroborant la faible présence d'eau dans le linge pressé. La localisation de la presse à l'entrée de l'atelier de Stephanus et la position de sa représentation sur le pilier de L. Veranius Hypsaeus confirment qu'il s'agit de la dernière étape du processus de foulerie, avant la rétrocession des vêtements - secs - à leur propriétaire.

L'inutilité de la fosse mise au jour dans l'atelier III, 10 d'Herculanum dans le cadre du nettoyage des vêtements - que l'on insère la presse dans la chaîne opératoire de la foulerie ou que l'on dresse une comparaison avec le seul exemplaire étudiable archéologiquement - rend à elle seule caduque l'interprétation du pressoir qui y a été découvert ${ }^{21}$. Dès lors, les canaux creusés dans la maie et la fosse associée désignent un instrument conçu pour extraire des liquides, probablement une presse à huile.

Cette réinterprétation pose problème. En effet, qu'il s'agisse de représentations iconographiques ou de vestiges archéologiques, les exemplaires de pressoirs à huile urbains attestés n'utilisent pas de vis mais des coins. Techniquement et formellement, la presse d'Herculanum apparaît bien éloignée des modèles couramment utilisés et représentés dans la région pour presser des olives en vue d'une production cosmétique. Pourtant, le canal circulaire incisé sur les maies en pierre - qui s'oppose aux canaux parallèles du pressoir herculanéen - ne renvoie qu'à l'utilisation de scourtins pour contenir la pâte d'olives devant être pressée. Cette technique n'est pas la seule attestée. Les textes du $\mathrm{I}^{\mathrm{er}} \mathrm{s}$. ap. J.-C. signalent l'utilisation d'un coffre en bois, appelé galeagra par Héron d'Alexandrie (Mec. 3, 16-17) ou

18 Les jumelles sont enfoncées dans le sol de $50 \mathrm{~cm}$ et ont une section de $30 \mathrm{~cm}$ par $50 \mathrm{~cm}$ (NSc 1912, p. 248-249). Seule l'empreinte de celle située au sud a été moulée.

19 Une première mention de ce creusement a été donnée par M. Della Corte (NSc 1912, p. 249).

20 Mart. 2, 43, $6:$ :...] sic tua suppositis collucent prela lacernis $[. .$.$] ; " De même tes presses brillent de tes manteaux super-$ posés [...] » ; Mart. 11, 8, 5 : [...] de Palatinis dominae quod Serica prelis [...] ; « [Des senteurs] de la soie sortie des presses de la maîtresse du Palatin [...] ».

21 Il serait par ailleurs inutile de gloser sur les risques de donner de faux plis disgracieux au linge à cause de la présence de canaux incisés dans la maie. 


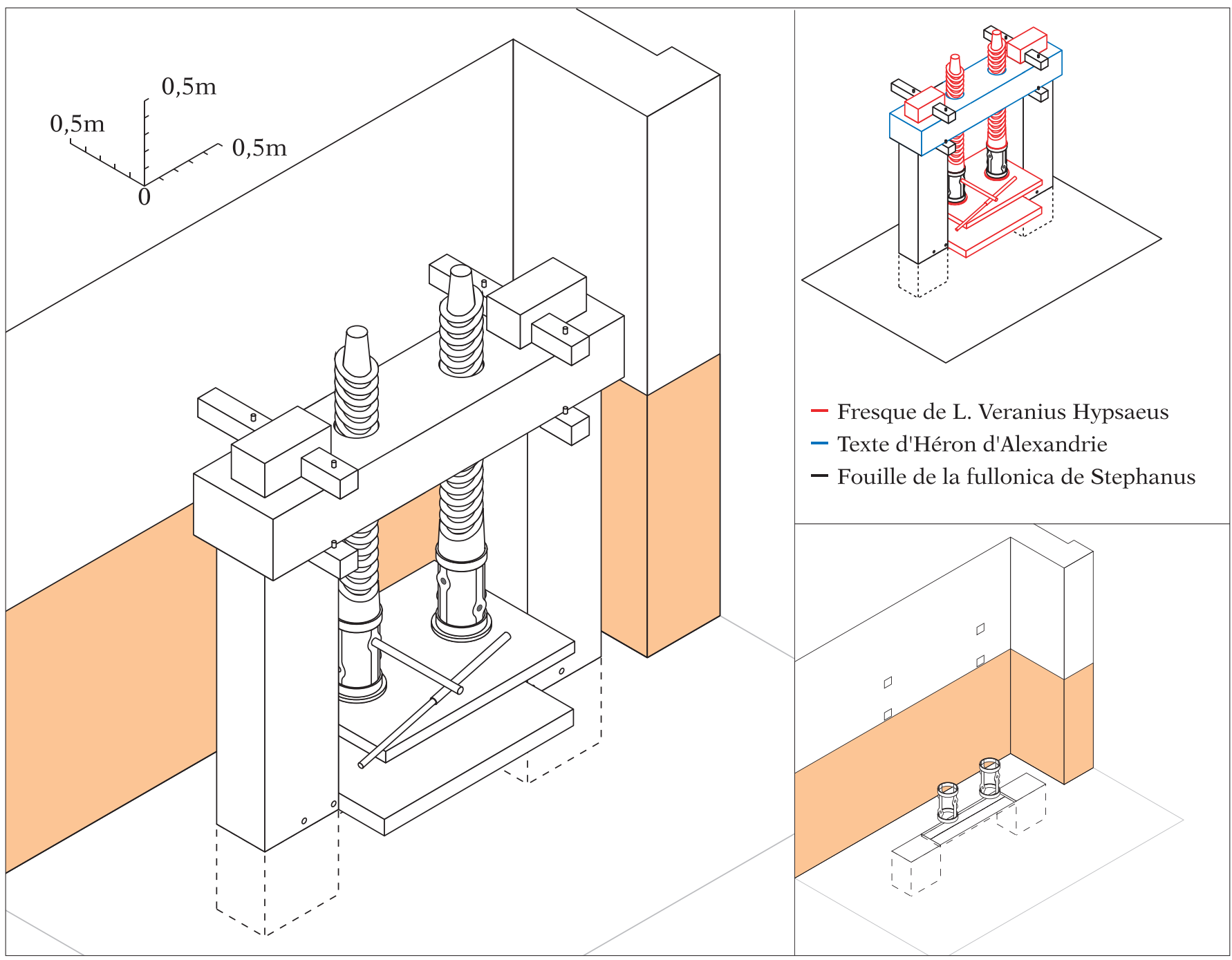

Fig. 7. Restitution axonométrique de la presse de la fullonica " de Stephanus » à Pompéi (I 6, 7). En encadré, en haut, justification de la restitution ; en bas, éléments de la presse mis au jour lors de la fouille (échelle de l'isométrie: 1/40 ; de l'encadré: 1/100) (dessin N. Monteix)

regulae par Pline l'Ancien (N.H., 15, 5) et Columelle. Ce dernier indique qu'il s'agit d'un système utilisé dans certaines régions, mais qu'il recommande pour les olives vertes ou pour extraire l'huile à parfum $(R . R ., 12,52,10$ et 12, 52, 10). Dans l'état actuel de la presse d'Herculanum, il est impossible qu'un coffre ait pu être employé car la maie et le plateau, de dimensions identiques, sont en contact direct avec les jumelles. En comparant les mesures données par les GSE avec celles que l'on peut prendre directement sur le pressoir restauré, une divergence de $20 \mathrm{~cm}$ apparaît : le banc a nécessairement été raccourci lors du remontage ${ }^{22}$. Nous pouvons dès lors proposer une restitution qui tienne compte des données transmises par les journaux de fouille et par les restes - mutilés - conservés in situ (fig. 8). L'écartement des jumelles, qui correspond à la longueur du banc, laisse une distance de $10 \mathrm{~cm}$ de chaque côté du plateau et de la maie telle qu'elle est conservée actuellement. Cet espace est largement suffisant pour insérer un coffrage en bois constitué par un assemblage de planches formant une cage (galeagra), conforme aux prescriptions

$22 \mathrm{Ce}$ raccourcissement du banc pourrait s'expliquer par l'état de la presse : en l'absence de sa partie inférieure, il a été nécessaire d'appuyer la maie contre les jumelles pour éviter de trop nombreuses intégrations ou pour rendre l'ensemble structurellement plus cohérent. Sur les problèmes de lecture des GSE quant à l'écartement des jumelles, cf. supra note 8. 


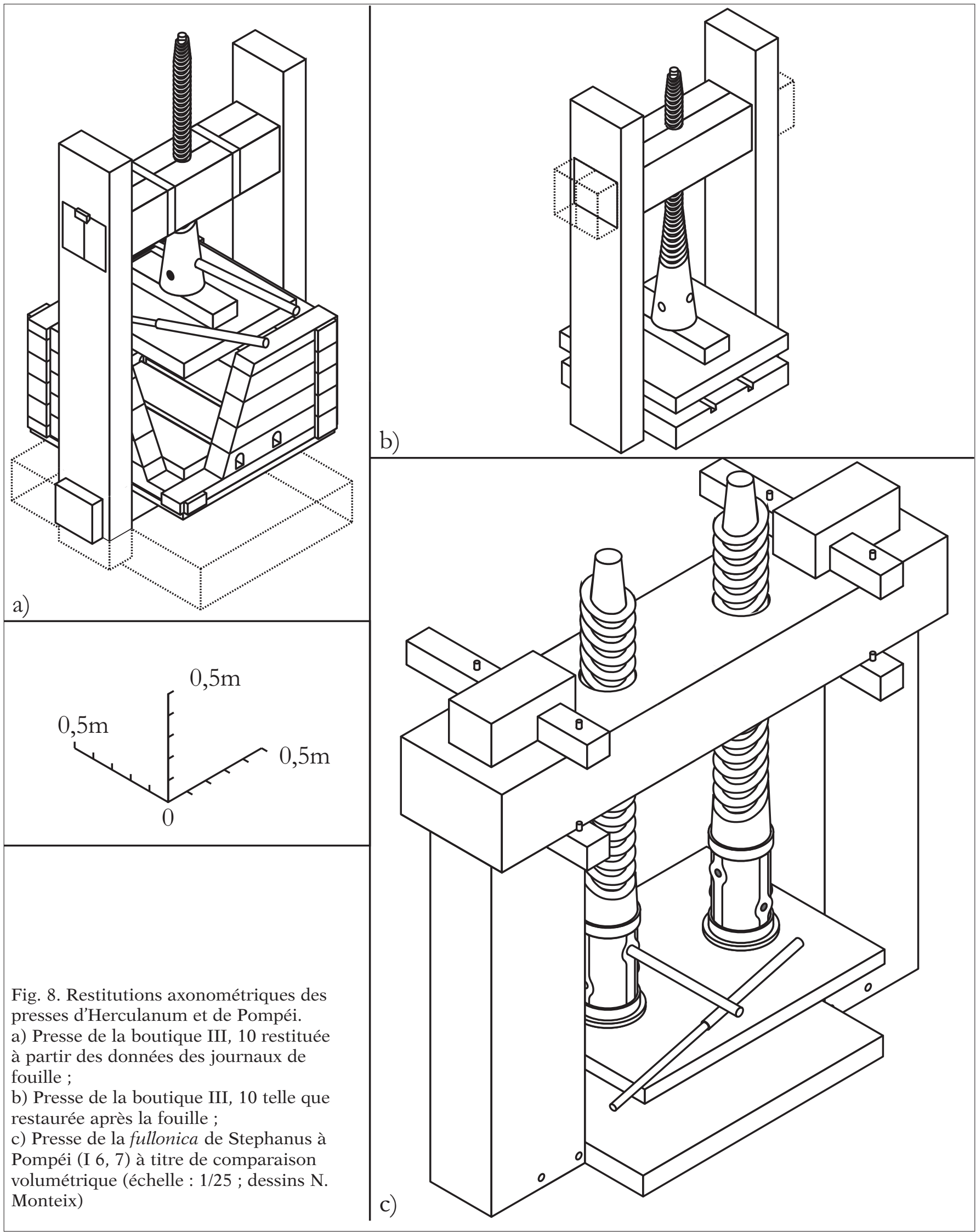


d'Héron d'Alexandrie, à la largeur des planches employées près ${ }^{23}$. Par ailleurs, il nous faut également restituer une seconde planche placée sous la maie de façon à ce qu'elle puisse supporter tant la cage que la maie. Une traverse serait également nécessaire pour former un cadre fermé. Les produits extraits - huile et margines - seraient réceptionnés dans la fosse située sous la presse, afin de les séparer. Au cours du pressage, l'huile coulerait ainsi, à travers les fentes du coffrage, mais également par le biais des canaux incisés dans la maie. Cette utilisation d'une fosse à la place d'un récipient mobile pour la décantation est notamment attestée dans une huilerie de Jordanie ou dans un exemple moderne ${ }^{24}$.

Nous pouvons donc abandonner définitivement l'interprétation proposée par A. Maiuri concernant la presse située en III, 10 : bien qu'elle fonctionne à vis, rien ne la lie avec l'artisanat textile. Elle ne peut pas supporter la comparaison avec les autres presses à vêtements identifiées à Pompéi, directement associées à des fouleries. De plus, la faible quantité d'eau vraisemblablement contenue dans le linge ne nécessite ni canaux d'écoulement, ni fosse de récupération ${ }^{25}$. En revanche, nous pouvons revenir à la première idée proposée par A. Maiuri : il se serait agi d'un instrument destiné à extraire de l'huile. Au-delà des aspects techniques, cette interprétation est également soutenue par les textes du $\mathrm{I}^{\text {er }} \mathrm{s}$. ap. J.-C. : bien que présenté comme une nouveauté, ce type d'appareil y est décrit.

Avec une telle presse, le local III, 10 devient une huilerie, voire un atelier de parfumerie. L'absence de toute structure pour broyer les olives n'est pas gênante : d'une part ce local a été fortement dégradé par les tunnels des fouilleurs du XVIII ${ }^{e}$ s., d'autre part les préparations des parfumeurs (huile, aromates, etc.) ne nécessitaient pas de moulins. Le plus souvent le broyage était effectué au mortier. L'absence de fourneau maçonné pour l'enfleurage ne peut constituer une objection car la peinture de la Casa dei Cervi (fig. 3) montre qu'on utilisait aussi bien des fourneaux mobiles, en fer, supportant un chaudron. En tout état de cause, cette interprétation s'accommode mieux avec la petitesse du local qui rend difficile les manipulations multiples des huiles et margines, nécessaires dans une huilerie tournée vers l'alimentation ou le combustible d'éclairage ${ }^{26}$.

\section{Pompéi}

Les auteurs antiques ne mentionnent pas spécifiquement Pompéi comme un centre de production de parfum, mais quatre inscriptions (deux électo- rales, une dédicace et un graffito) citent des parfumeurs soit collectivement, soit individuellement : les unguentarii prennent partie pour Verus et Modestus lors des élections (C.I.L. IV, 609 et 9932a ; Giordano et Casale 1982, p. 7), le parfumeur Phoebus était client du lupanar (C.I.L. IV, 2184) et M. Decidius Faustus fit une dédicace dans le temple d'Apollon (C.I.L. X, 892). Dès 1979, W. Jashemski a attiré l'attention sur l'importance des cultures florales dans la ville même et sur la fabrication locale des parfums. Les jardins de la Casa del giardino di Ercole et de la maison de Pansa notamment auraient été cultivés en espèces florales destinées à la confection de guirlandes et de parfums. Elle proposait d'identifier la presse découverte en 1927 dans les boutiques VII 4, 24-25, située via degli Augustali, comme celle d'un parfumeur (fig. 8). En effet une huilerie placée si près du forum serait incongrue et sa localisation est proche du lieu de découverte des deux inscriptions électorales commanditées par les unguentarii (Jashemski 1979, p. 276). D. Mattingly a développé cette hypothèse en rapprochant le type de presse de celui connu par les quatre peintures de Pompéi et d'Herculanum représentant des appareils à coins utilisés par des parfumeurs. Il a proposé de restituer non une presse à vis, comme Maiuri avait fait reconstruire, mais une presse à coins, seul appareil attesté par les sources et l'iconographie se rapportant aux parfumeurs (Mattingly 1990).

Cette probable parfumerie n'était pas isolée : un autre pressoir existait dans une des boutiques de la

23 Héron (Mec., 3, 16-17) donne deux modes de construction pour ce coffrage. La seule différence entre notre restitution et ses indications tient à l'épaisseur des planches. Selon lui, il est nécessaire de prendre des planches épaisses de 6 doigts de 19,3 mm, soit environ 11,5 cm d'épaisseur. Dans le cas de la presse trouvée en III, 10, l'espace disponible entre la maie et les jumelles est limité à $10 \mathrm{~cm}$. Les données de Héron ne sont de toutes façons qu'indicatives et pour une petite presse du type de celle d'Herculanum des planches de moins de $10 \mathrm{~cm}$ d'épaisseur suffisaient.

${ }^{24}$ L'huilerie de Khirbet Edh-Dharih (Jordanie), date du I ${ }^{\text {er }}$ ou du $\mathrm{II}^{e}$ s. ap. J.-C. et dispose de deux pressoirs à levier et de deux fosses de décantation (Villeneuve 1988, p. 464). Une gravure sur cuivre réalisée par Johannès Stradan $\left(\mathrm{XVI}^{\mathrm{e}}\right.$ s.) montre une presse à vis utilisant des scourtins et une fosse de décantation (cf. Amouretti et alii 1984, fig. 1, p. 383).

25 Ajoutons à cela que si cette fosse n'avait contenu que de l'eau, aucune matière carbonisée n'y aurait été retrouvée.

26 Certains des points délicats dans cette interprétation ne pourront jamais être éclaircis, vues les destructions occasionnées par les premiers fouilleurs du site. En revanche, une nouvelle mise au jour de la fosse de décantation permettrait certainement de confirmer l'hypothèse faisant de cette presse une presse à huile. 


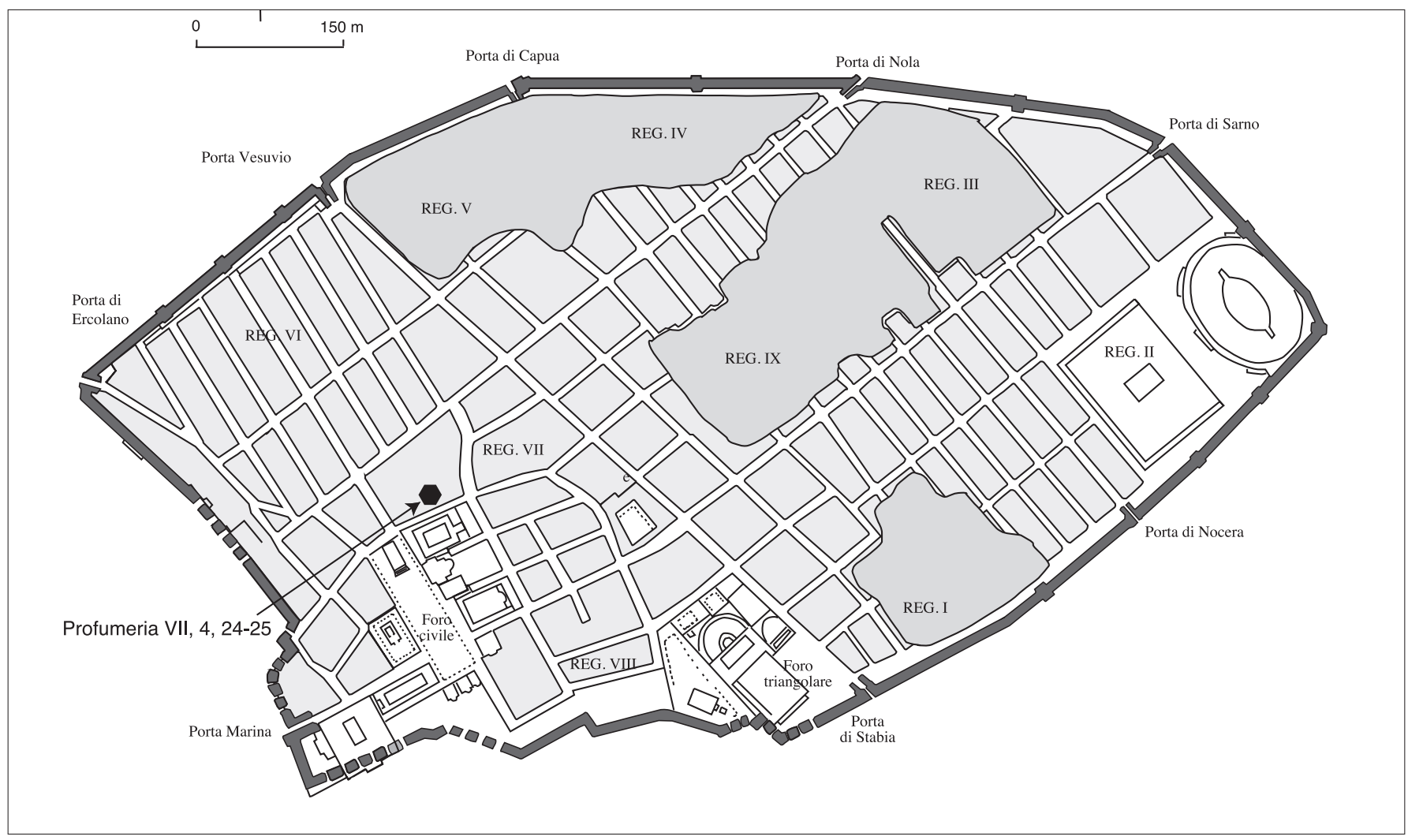

Fig. 9. Localisation de la parfumerie VII, 4, 24-25 à Pompéi (dessin M. Pierobon)

rue (la maie se trouve aujourd'hui dans la maison d'Ariane). Dans la via degli Augustali, le 3 octobre 1823, «nel disterrarsi una bottega posta a destra la strada al lato settentrionale del Foro civile si rinvennero, i seguenti oggetti. Bronzo... una coppa grande di bilancia, due coppe di bilancia, due simili più piccoli, un asta appartenente ad esse bilance, altra simile asta, ...uno strigile. Vetro. Una piccola carafina..., nove bocce a palla sul collo stretto, di diverse misure, altre trenta più piccole carrafine, molte delle quali rotte nell'orificio. Quattro bottiglioni grandi a palla e col collo stretto, ... due vasi di figura circolare..., altra boccia rotta..., 215 carafine, chiamate comunemente lacrimatoj, la maggior parte della quali rotte nell'orificio. Terracotta... un mortajo per salsa col suo pestello... Marmo, tre mortaj di diversa grandezza, uno dei quali ha il suo pistello, 6 pesi di varie grandezze...». Cette boutique contenait donc outre toutes sortes d'objets en or, argent, bronze (nombreux vases) et fer, un grand nombre de bouteilles de verre et de flacons à parfum, ainsi qu'un attirail compatible avec la prépara- tion des parfums : plusieurs balances, trois mortiers en marbre et un en céramique, six poids (Fiorelli 1860-1864, II, p. 82-83). Par ailleurs, lors de dégagements effectués le 27 février 1829 en présence du Roi de Bavière dans une boutique située devant les thermes du Forum, en VII 5, 29, on trouva 550 "carafinette", 11 "unguentarii" et 55 "boccettini" (Fiorelli 1860-1864, II, p. 219). Il semble donc se dessiner, dans la via degli Augustali et la via del Foro, une concentration d'ateliers et de boutiques de parfumeurs évidemment favorisée par la proximité du forum et des thermes.

\section{Une parfumerie ensevelie en 79 après J.-C.}

Les boutiques de VII 4, 24-25 furent dégagées à partir de mai 1822. Selon G. Fiorelli, il s'agit de deux boutiques contiguës séparées par une cloison de bois. "Elles formaient la partie antérieure de l'habitation du boutiquier qui consistait surtout en un atrium tuscanicum décoré de diverses peintures, mais dont subsistait un panneau à fond monochrome rosâtre, représentant Hercule ivre qui traîne 
Augé, entouré d'un groupe de faunes emmené par Silène (Corallini 2001, p. 201-202, P083). Ce dernier qui porte sur l'épaule la massue du héros, urine sur ses jambes. Dans cet atrium, outre la margelle de la citerne et un escalier, se trouvaient le triclinium et une chambre, séparés par le couloir menant à la cuisine. Cette dernière, relativement grande, comportait un foyer au-dessus duquel était peint un Genius sacrifiant entre les deux lares. Les latrines, un escalier pour monter à l'étage et à gauche deux pièces appartenant peutêtre à un édifice antérieur complétait le tout. » (Fiorelli 1875, p. 216).

Le pressoir situé dans la boutique 25 n'est pas signalé dans ce texte car il a été dégagé en 1927, avant la restauration des parties en bois (fig. 10). "L'indagine portò alla scoperta, in corrispondenza di due piedritti in laterizio, di una vaschetta o pozzetto quadrangolare (m. 1,65 x $0,90 \times 0,64)$ entro il quale i frammenti della base in travertino apparivano precipitati e rotti alla rinfusa. Procedutosi con la maggiore accuratezza possibile e con la più diligente osservazione di tutti i relitti del terreno allo scrostamento del pozzetto, si notarono gli avanzi chiari ed evidenti di una robusta armatura lignea, carbonizzata per lenta trasformazione organica, sulla quale veniva a poggiare il piano inferiore del pesante parallelepipedo in travertino. Dell'armatura in legno restavano i tronconi di due robustissime travi disposte ed incastrate a croce e al disopra di queste travi due piani lignei, dei quali l'uno era circolare (dello spessore di m. 0,09, l'altro quadrangolare (dello spessore di m. 0,11), attraversa ambidue in correspondenza del punto d'innesto delle sottostanti travi, da un solido perno cilindrico in ferro (lungezza m. 0,32) ; infine si osservò all'estremità del troncone più lungo delle travi a croce, la presenza di appositi fori per l'incastro dei piedritti in legno della armatura superiore del torchio, l'esatta altezza di questi piedritti era data dalla conservazione di un alveolo del trave superiore del telaio su uno dei piedritti in muratura del muro orientale della bottega.... » (Maiuri 1928-1929, p. 517-518).

La construction du pressoir et le remodelage des boutiques sont postérieurs au tremblement de terre de 62 ou 63. Les fouilles effectuées en 2001-2002 ont découvert, dans le sol de béton de tuileau couvrant l'ensemble des deux boutiques, trois monnaies dont deux identiques datées de l'année 65 après J.-C., volontairement déposées dans la chaux. Il a donc fallu au moins deux ou trois ans pour procéder à la reconstruction de la boutique et de la maison. Ce délai est tout à fait normal en ces circonstances ; il est même court si l'on considère que les monnaies forment un dépôt de fondation destiné à dater la réfection de la

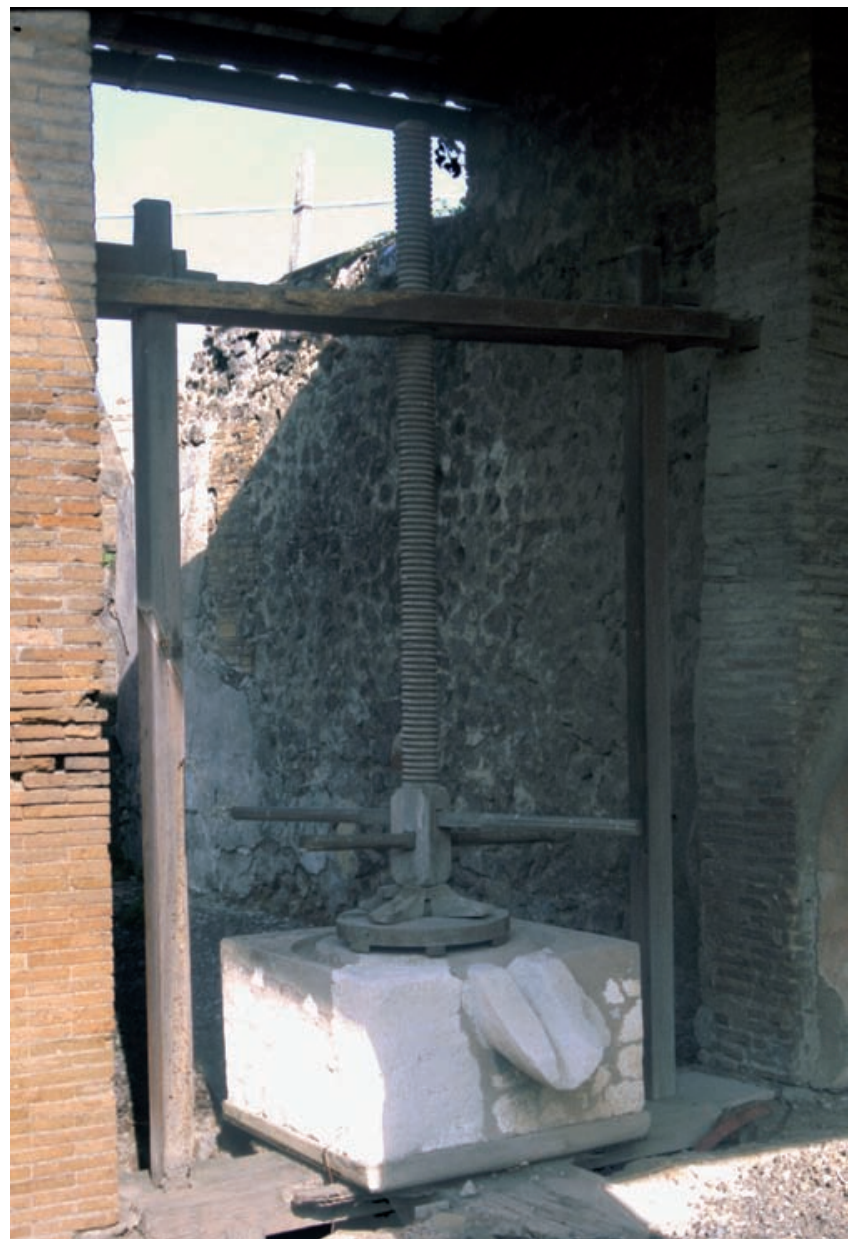

Fig. 10. Pressoir à vis reconstitué par A. Maiuri dans la boutique VII, 4, 24-25 (cliché J.-P. Brun)

boutique. Jean Andreau a montré en effet que peu de maisons avaient été reconstruites avant 70, mais que les boutiques et les ateliers avaient été les premiers réparés. À Lisbonne après le séisme de 1755, la reconstruction dura vingt ans, mais ce sont aussi les boutiquiers et les artisans qui furent les plus prompts à faire les travaux afin de ne pas perdre leur clientèle et de continuer leurs affaires (Andreau 1973).

Les fouilles de 2001-2002 n'ont rien trouvé qui appartienne à la dernière phase d'occupation (fig. 11), car les dégagements du XIX ${ }^{\mathrm{e}}$ siècle et les fouilles de 1927 n'ont laissé subsister aucun niveau contemporain de l'éruption (Fiorelli 1860-1864, II, p. 52-54 et 79-80). Pour nous les boutiques sont vides à l'exception du pressoir. Ce dernier, malgré la description d'A. Maiuri citée ci-dessus, reste mal connu. En effet, les travaux effectués en 1927 ont détruit 
une bonne part des constructions romaines. La seule certitude est que la fosse quadrangulaire maçonnée a livré deux poutres croisées qui supportaient deux plateaux de bois, l'un circulaire épais de $9 \mathrm{~cm}$, l'autre carré épais de $10 \mathrm{~cm}$. Toutes ces pièces étaient assujetties par une fiche en fer longue de $30 \mathrm{~cm}$. Aux deux extrémités de la poutre longitudinale étaient encastrés deux montants dont la section n'est pas donnée. Selon A. Maiuri, le plateau circulaire et l'axe de fer auraient servi à faire tourner la maie et le pressoir aurait été actionné par une vis. C'est d'ailleurs sur ce schéma qu'il fit réaliser une reconstitution. Il est très douteux que la maie du pressoir ait été mobi- le, vu son poids ; la faire tourner était d'ailleurs inutile puisqu'on changeait les récipients placés sous le bec. Quant à restitution de l'instrument, aucun indice parmi la description des bois carbonisés ne permet de savoir s'il s'agissait bien d'une vis. David Mattingly, se fondant sur les représentations de pressoirs à coin des peintures de Pompéi et d'Herculanum, a proposé de restituer plutôt un appareil de ce dernier type. La position de l'engin entre deux piliers de briques libère ses faces antérieures et postérieures et autoriserait donc des mouvements de frappe des coins à l'aide de maillets. À la différence de l'exemple de Paestum que nous exami-

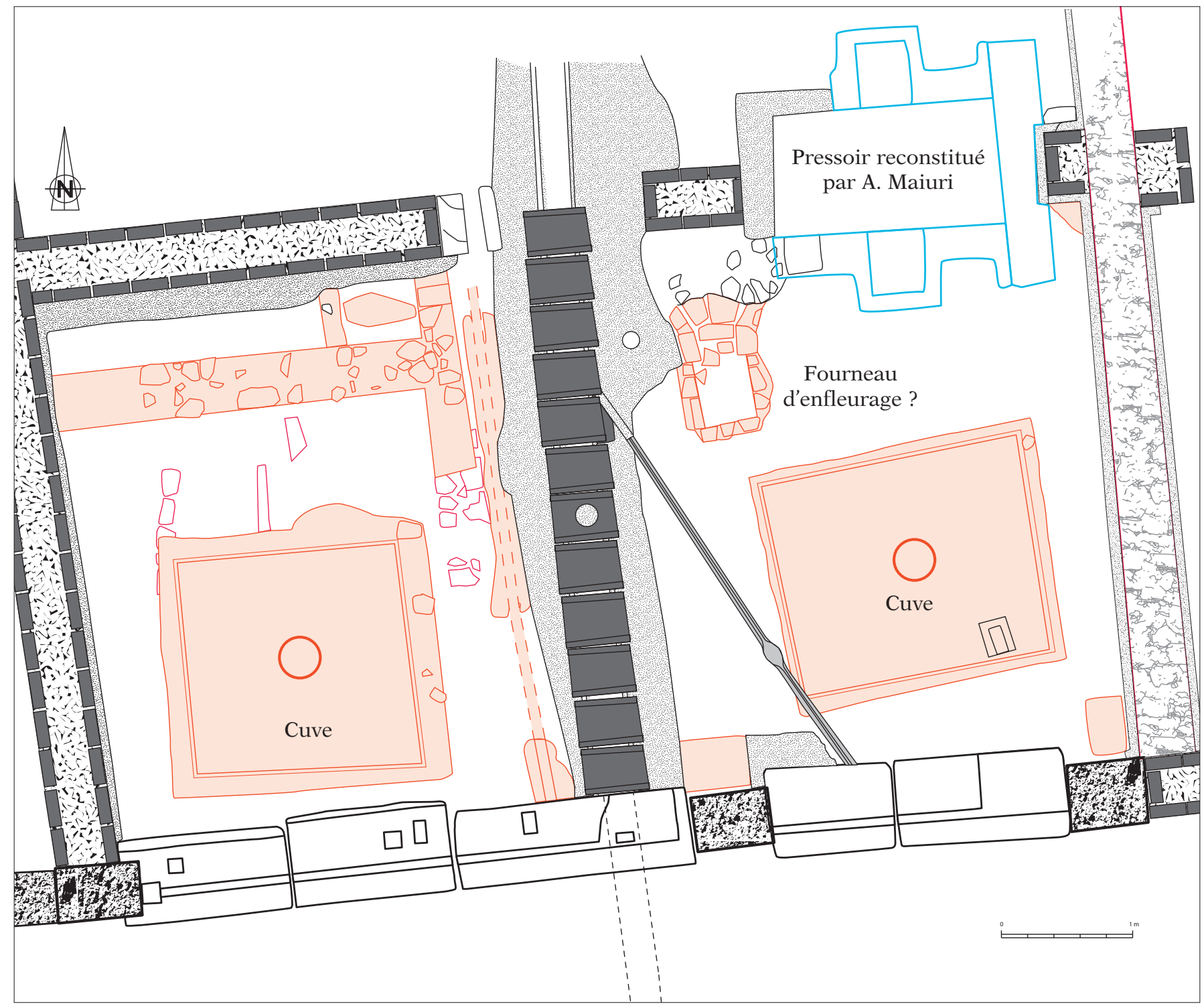

Fig. 11. Plan de la boutique VII, 4, 24-25. En rouge: phase antérieure au tremblement de terre de 62 ou 63 après J.-C. En noir : état en 79. En bleu : reconstruction de 1927 (plan J.-P. Brun) 
Fig. 12. Pompéi VII 4, 24-25 : état de la boutique avant 62 ou 63 de notre ère.

Au premier plan à gauche, alandier d'un fourneau.

Au centre, cuve.

(Cliché J.-P. Brun)

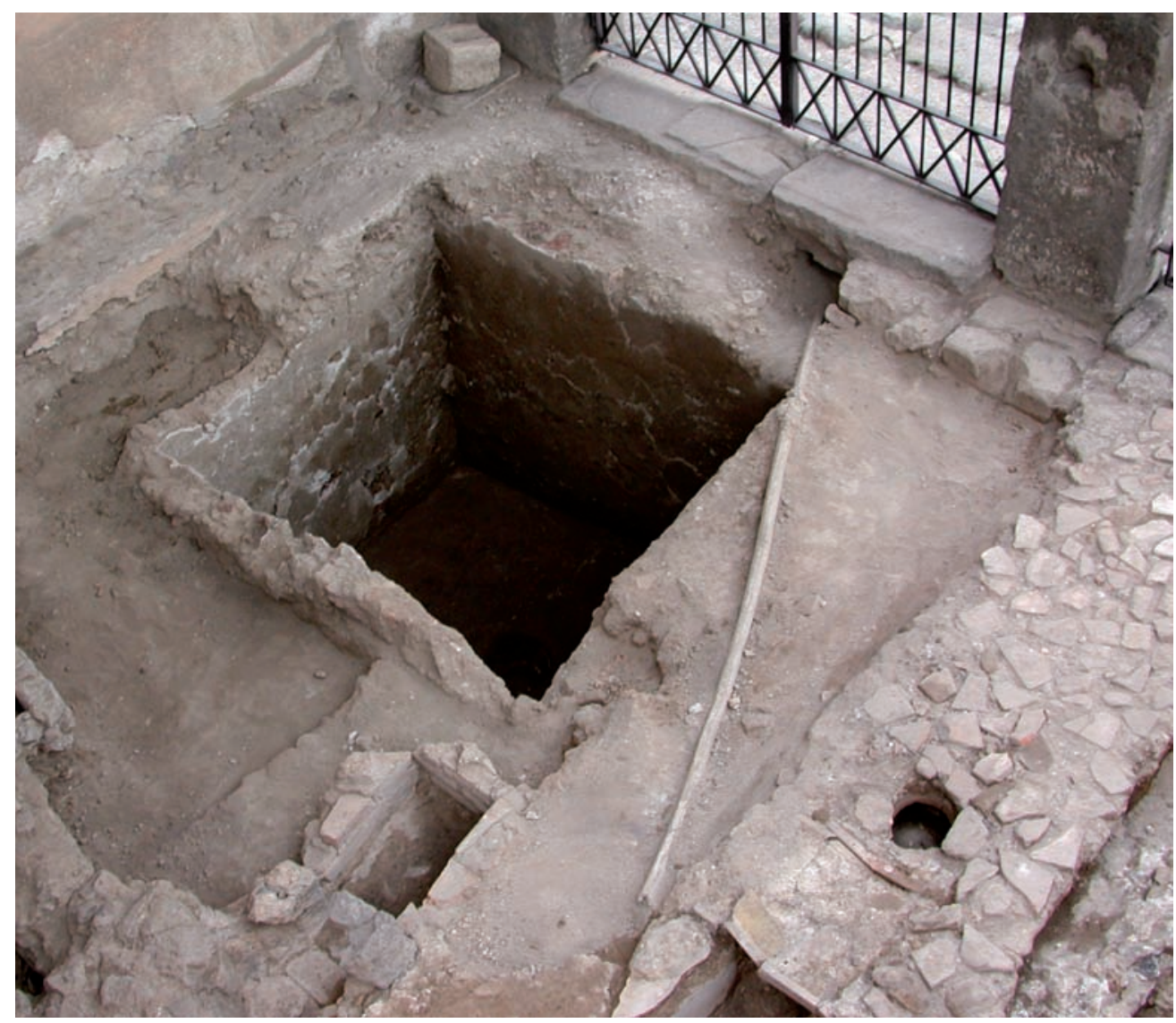

nons ci-après, une telle reconstitution est compatible avec les vestiges préservés. Toutefois, l'exemple du pressoir à vis d'Herculanum incite à la prudence : dans les années qui suivent le tremblement de terre, les appareils à vis, bien plus pratiques et efficaces, étaient disponibles. La reconstruction des lieux de production fut certainement l'occasion d'adapter les instruments de travail à de nouvelles techniques et le pressoir de la boutique VII 4, 24-25 a pu fort bien être analogue à celui de la boutique d'Herculanum.

\section{Une parfumerie antérieure au tremblement de terre?}

La phase antérieure au tremblement de terre est mieux connue. En effet, sous le sol en mortier de tuileau posé lors de la reconstruction de la boutique, deux cuves et une chaudière ont été découvertes (fig. 11 et 12). Les deux cuves mesurent 1,70 m par $1,60 \mathrm{~m}$ et $1,80 \mathrm{~m}$ par $1,60 \mathrm{~m}$; leur profondeur peut être estimée à $1,50 \mathrm{~m}$ environ et leur contenance moyenne à 4300 litres. Leur fond est en béton de tuileau et comporte une cuvette de puisage formé d'un fond d'amphore. Les parois sont recouvertes intérieurement d'un enduit de chaux dont la partie basse repose sur la dalle du fond. Les analyses chimiques effectuées par Nicolas Garnier ont mis en évidence la présence d'acides gras végétaux (palmitique et stéarique) et de stérols végétaux qui peuvent résulter de la décomposition d'huile d'olive.

Entre les cuves, se trouve la base d'une chaudière ultérieurement détruite en partie par la construction du pilier en brique situé à l'ouest du pressoir. Elle comprend un alandier rectangulaire, une bouche aux montants en blocs de basalte et une chambre de chauffe. Le diamètre de la chaudière devait avoisiner $0,70 \mathrm{~m}$ et il faut certainement restituer en partie haute un chaudron métallique. La chaudière doit-elle être interprétée comme un fourneau d'enfleurage ? Elle aurait alors chauffé un récipient en plomb ou en cuivre rempli d'eau dans laquelle aurait été disposé un chaudron contenant l'huile et les aromates. Théophraste ( $O d .22)$ indique en effet que l'huile doit être chauffée au bain-marie et que le chaudron ne doit pas être en contact avec la flamme, car le parfum risquerait de prendre une odeur de brûlé.

Il faut souligner toutefois que la dimension des cuves ne paraît pas compatible avec une production 


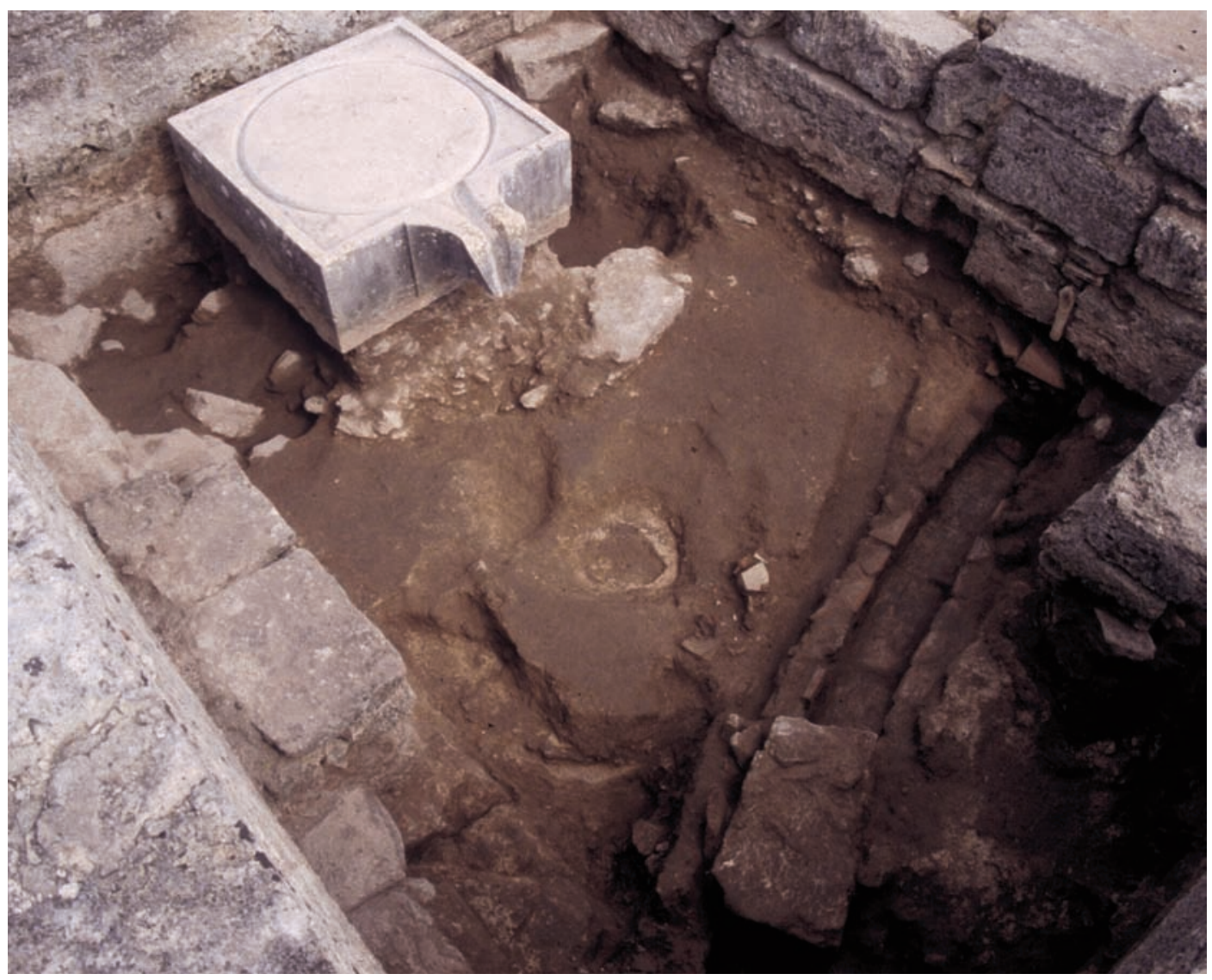

Fig. 13. Paestum, vue générale de la boutique située à l'angle nord-ouest du forum. La maie en marbre est flanquée de trous de poteaux pour les montants de la presse.

(Cliché J.-P. Brun) d'huile uniquement destinée aux parfums. Deux hypothèses se présentent donc. Soit la boutique abritait une parfumerie et la chaudière servait de fourneau d'enfleurage ; soit ces vestiges correspondent à une huilerie "traditionnelle" qui, vu sa localisation, travaillait peut-être préférentiellement pour les parfumeurs voisins, mais qui faisait aussi des huiles alimentaires, voire des huiles lampantes. Dans ce cas, la chaudière aurait servi à chauffer l'eau nécessaire aux pressées successives.

\section{Paestum}

Dans les années 1930, A. Maiuri fit dégager le côté nord-ouest du forum de Paestum. À cette occasion, furent mises au jour les boutiques qui bordaient cette partie de la place. L'une d'elles livra une maie de pressoir immédiatement interprétée comme faisant partie d'une huilerie. En 1995, grâce à la générosité d'Emanuele Greco, il a été possible de reprendre la fouille de ce secteur et de donner corps à l'hypothèse attribuant ce pressoir non à une banale huilerie, qui aurait été peu à sa place sur le forum, mais à une parfumerie (fig. 13). La fouille a été publiée en détail dans les Mefra 1996. Les acquis en sont résumés ci-après et l'interprétation du système de pressurage est ensuite revue.

La boutique qui mesure 4,30 $\mathrm{m}$ de longueur et 3,50 m de largeur est accolée au mur occidental du forum. Une première boutique a été construite en opus quadratum sur des niveaux de rues qui remontent à l'époque classique et vraisemblablement à l'époque archaïque. Le mobilier céramique et les monnaies trouvés dans les couches de construction sont datables des $\mathrm{IV}^{\mathrm{e}}$ et $\mathrm{III}^{\mathrm{e}}$ siècles avant J.-C. montrant que la boutique remonte à la création du forum de la colonie déduite en 273 avant J.-C. Le sol alors établi fut percé par la suite de fosses contenant entre autres des fragments de flacons à parfums. Ce pourrait être un indice que, dès les origines, cette partie du forum était consacrée au commerce des huiles parfumées.

Cette boutique a été utilisée durant environ trois siècles puis a été remodelée vers le milieu du ${ }^{\text {er }}$ siècle après J.-C. (ou peu après) en remployant les blocs d'opus quadratum liés à la chaux et comportant des bouchons de tuiles, pour reconstruire les 
murs périmétraux (état 3 ). Au cours de la phase suivante (état 4) datable de la période flavienne ou du début du $\mathrm{II}^{\mathrm{e}}$ siècle, le pressoir fut mis en place ; il semble contemporain de la pose d'un sol en opus signinum dont seuls quelques lambeaux sont conservés. Ce sol fut traversé par un égout dans le courant du $\mathrm{II}^{\mathrm{e}}$ siècle (état 5 ) et la boutique équipée du pressoir a été utilisée jusqu'au $\mathrm{III}^{\mathrm{e}}$ siècle. Sa destination change au $\mathrm{IV}^{\mathrm{e}}$ siècle car le pressoir est alors démantelé. Un nouveau mur de façade, en gros blocs de tuf de remploi, est construit et l'intérieur de la pièce est fortement remblayé jusqu'à recouvrir la maie.

Les niveaux des états 3 à 5 ont été détruits par les fouilles anciennes mis à part quelques lambeaux dans lesquels ont été découverts trois unguentaria en céramique de la fin du $\mathrm{I}^{\mathrm{er}}$ siècle avant J.-C. - début du $\mathrm{I}^{\mathrm{er}}$ s. après J.-C. et des fragments de balsamaires en verre des $\mathrm{I}^{\mathrm{er}}$ et $\mathrm{II}^{\mathrm{e}}$ s. après J.-C.

La datation du pressoir vers la fin du ${ }^{\text {er }}$ siècle de notre ère exclut qu'il ait pu servir à une production agricole, car le forum remplit alors pleinement sa fonction de place publique et de marché. La qualité du matériau employé et de la taille font de la maie autant un instrument de travail qu'un élément de décoration : c'est un bloc de marbre blanc mesurant 1,49 $\mathrm{m}$ de long, soit 5 pieds, $1,19 \mathrm{~m}$ de large, soit 4 pieds et $0,48 \mathrm{~m}$ de haut (1 pied et 10 doigts). La face supérieure présente un carré de 1,19 m de côté formé par une plate-bande en saillie de 1,8 cm. Au centre, un cercle de $0,98 \mathrm{~m}$ est entouré d'une rigole à section en $\mathrm{V}$ profonde de $2,8 \mathrm{~cm}$. Cette rigole s'élargit et d'approfondit au niveau d'une saillie de la pierre qui forme un bec verseur. Il n'existe aucune trace de cuves de décantation bâtie qui, malgré les destructions des fouilles anciennes, auraient laissé des vestiges dans le sol. Le liquide devait donc être recueilli dans des bassins mobiles, peut-être en métal comme on le voit sur les peintures de Pompéi représentant les parfumeurs au travail.

De l'appareillage en bois qui était fixé à la maie, deux traces ont été conservées. À l'ouest de la maie se trouvait le calage d'un poteau vertical mesurant $45 \mathrm{~cm}$ par $30 \mathrm{~cm}$ de section enfoncé dans le sol d'environ $1 \mathrm{~m}$ par rapport à la surface de la table de presse (fig. 14). Son

Fig. 14. Paestum : plan de la parfumerie (plan J.-P. Brun) pendant qui devait être placé de la même manière à l'est de la pierre a été détruit par les fouilles anciennes, trop poussées dans cet angle de la boutique. La deuxième trace est située sous la pierre. Alors que la maie repose sur un remblai compact d'éclats de taille, la zone centrale est formée de couches de colluvions très fines marquant l'emplacement d'une poutre qui accouplait les deux montants.

La présence de ces deux montants assure que le pressoir était constitué d'un cadre vertical enfoncé dans le sol et certainement fixé en partie haute dans les murs. Dans l'article publié dans les Mefra, étaient présentées deux hypothèses de restitution : pressoir à coins et pressoir à vis. Après discussion des deux possibilités, tout en laissant la question pendante, le choix avait été fait de restituer graphiquement un pressoir à coins, en se fondant sur trois arguments : les quatre peintures de Pompéi et d'Herculanum montrent des appareils à coins, le texte d'Héron

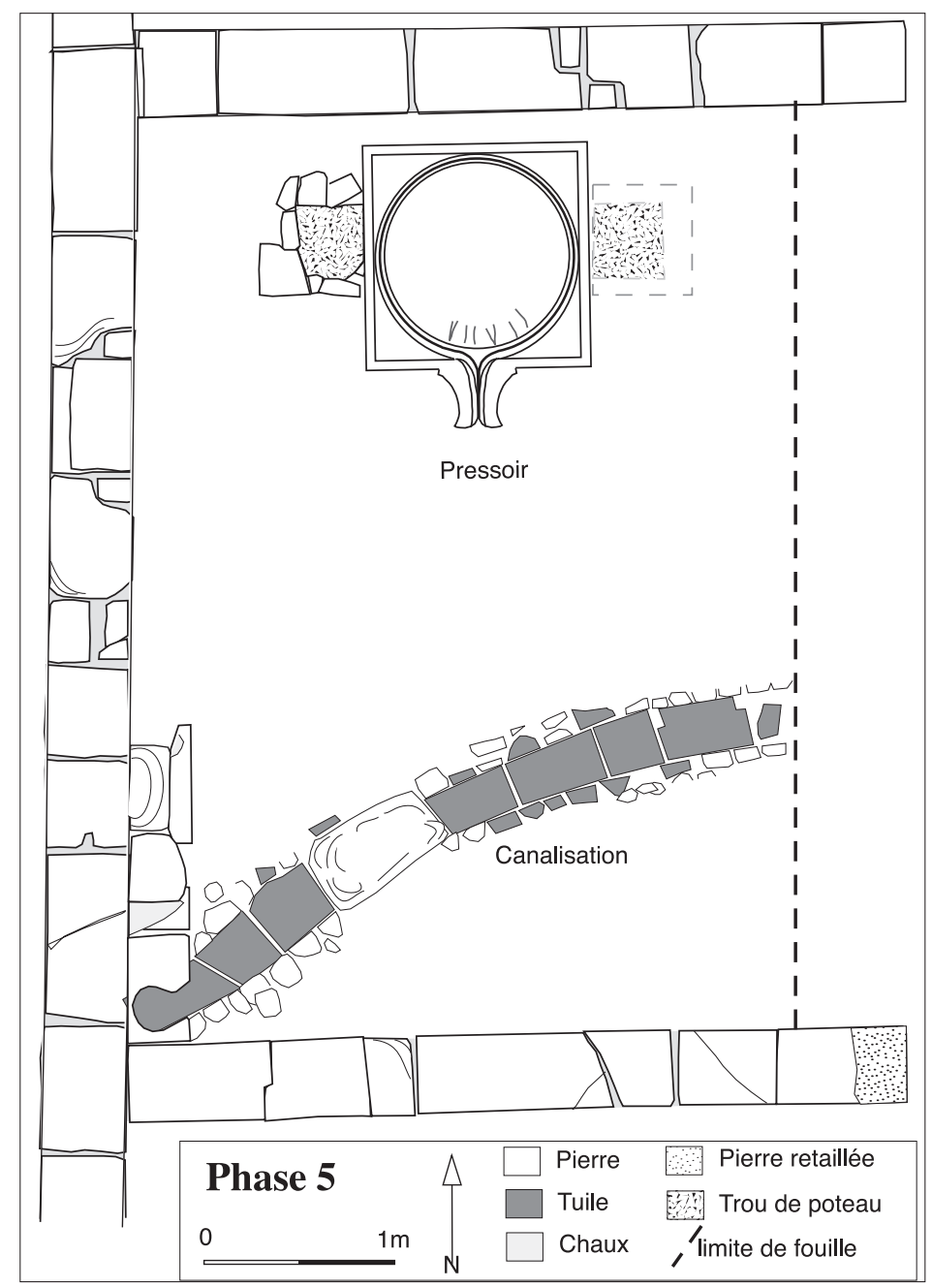




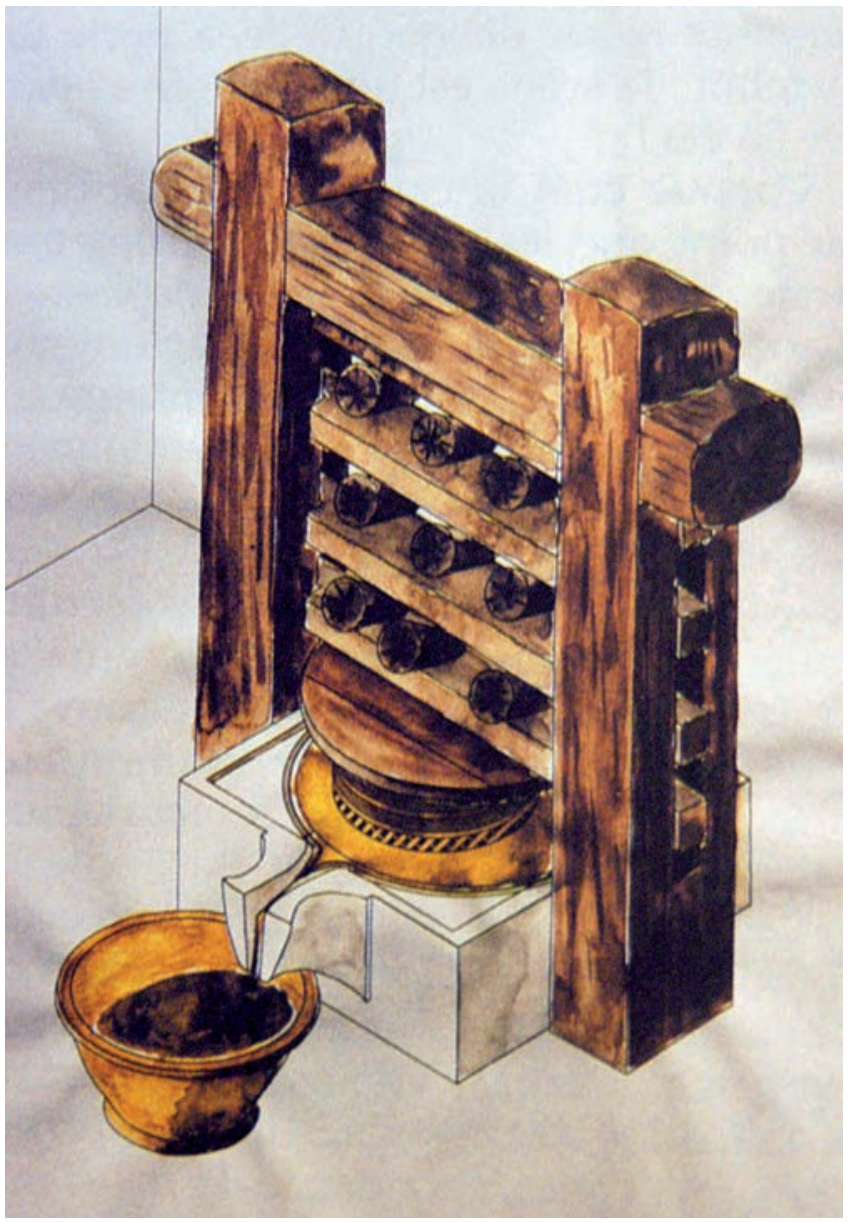

Fig. 15. Paestum : proposition erronée de restitution d'un pressoir à coins (dessin J.-P. Brun)

d'Alexandrie mentionne la presse à coin comme étant utilisée par les parfumeurs et aucune source ne cite l'utilisation d'instrument à vis par ces derniers (fig. 15). Il avait été bien vu que « le principal obstacle à une telle identification réside dans le manque d'espace entre le pressoir et le mur. Toutes les peintures montrent que l'on actionnait la presse en frappant alternativement sur la rangée de coins de la face antérieure et sur ceux de la face postérieure. Cette manœuvre est rendue quasiment impossible par la position de la presse... ». De fait, l'attribution de la presse à vis centrale d'Herculanum à la production des parfums lève tout obstacle à la restitution d'un appareil analogue à Paestum (fig. 16). Dans ce cas, la difficulté liée à la proximité du mur est levée et c'est donc à cette option qu'il faut se ranger.

\section{Représentations figurées et realia}

Les textes qui évoquent les parfumeurs au travail et leurs instruments sont très rares. Il s'agit principalement d'un passage des Mechanika de Héron d'Alexandrie qui n'est connu que par une traduction arabe (II, 43, 4). Après avoir décrit les pressoirs à levier et câble ou à vis et les appareils à une ou deux vis pressant directement la pâte d'olive, Héron écrit « la quatrième machine simple, qui suit les précédentes, est celle qui est appelée le coin. Elle sert dans quelques-unes des préparations des parfumeurs ». La traduction arabe est quelque peu inexacte si on en croit la citation que fait Pappos "La quatrième force est le coin; il rend de grands services dans les presses pour les parfumeries...» Une seule source évoque, vers le milieu du $\mathrm{I}^{\mathrm{er}}$ siècle de notre ère, un type de presse probablement traditionnel dans les parfumeries. Le texte est éclairé et renforcé par le témoignage stéréotypé des quatre peintures de Pompéi et d'Herculanum. Il se dégage donc une unanimité entre texte et iconographie qui semble ne laisser aucune place à d'autres appareils dans les parfumeries. Mais les témoignages sont très peu nombreux et tous datés vers le milieu du Ier siècle de notre ère. Qu'en était-il auparavant, qu'en fut-il par la suite ? Les installations de parfumeurs ont mis très longtemps à se dégager des cuisines et, pour les périodes anciennes, leur identification est très difficile. Toutefois, grâce à plusieurs bas-reliefs, nous savons qu'en Égypte, les parfumeurs utilisaient des pressoirs à torsion pour extraire les substances aromatiques et les huiles. Il est vraisemblable que ce procédé très simple, qui consiste à mettre les ingrédients dans un sac en tissu que l'on tord avec des bâtons, a été utilisé depuis une époque très ancienne et qu'il n'a cessé de l'être même lorsque les artisans ont adopté des appareils en bois, en commençant par les systèmes à coins. Les pressoirs à coins devaient exister en Grèce hellénistique, au moins vers la fin de la période, car les deux pressoirs de l'îlot IB du Quartier du stade de Délos ne peuvent correspondre qu'à ce type. En effet, dans cette installation datée de la fin du $\mathrm{II}^{\mathrm{e}}$ siècle avant J.-C. la fouille a retrouvé l'empreinte, dans le mortier, des montants de bois qui flanquaient les maies de marbre ; il s'agissait donc d'appareils verticaux, or, à cette période, la vis n'étant pas encore appliquée aux pressoirs, seul un pressoir à coins peut rendre compte des vestiges visibles. Cette phase technique a duré jusqu'au milieu du $\mathrm{I}^{\text {er }}$ siècle après J.-C. et c'est à elle que se rapportent les sources mention- 
nées plus haut. À partir de la seconde moitié du Ier siècle, les appareils à coins ont dû progressivement laisser la place aux pressoirs à vis centrale, plus aisément maniables. Ainsi à Paestum, sous les Flaviens, c'est un pressoir de ce type qui a certainement été mis en place.

Il y a donc sur ce point, comme sur bien d'autres se rapportant aux techniques, un certain péril à appliquer à l'ensemble de la période antique les sources textuelles et iconographiques écrites ou réalisées à un moment précis. Les techniques n'ont pas été statiques durant la période romaine ; elles ont intégré rapidement certaines inventions lorsqu'elles étaient compatibles avec les pratiques sociales et les disponibilités économiques des artisans. C'est le manque de sources, en un mot notre méconnaissance des techniques antiques, qui nous pousse à utiliser les rares éléments à notre disposition pour recréer une histoire statique que nous interprétons comme stagnation.

En 79, les techniques des parfumeurs avaient déjà changé par rapport aux représentations qu'on en donnait dans la peinture contemporaine qui, de plus, ne se voulait pas l'illustration fidèle des dernières pratiques mais une représentation idéalisée d'une réalité déjà surannée mais aisément identifiable par le spectateur. De même qu'aujourd'hui, le mortier et le pilon restent les emblèmes des pharmaciens bien qu'ils ne les utilisent plus guère, de même le pressoir à coins a dû continuer à symboliser l'activité des parfumeurs bien après qu'ils eurent adopté des appareils plus performants mais moins spécifiques.

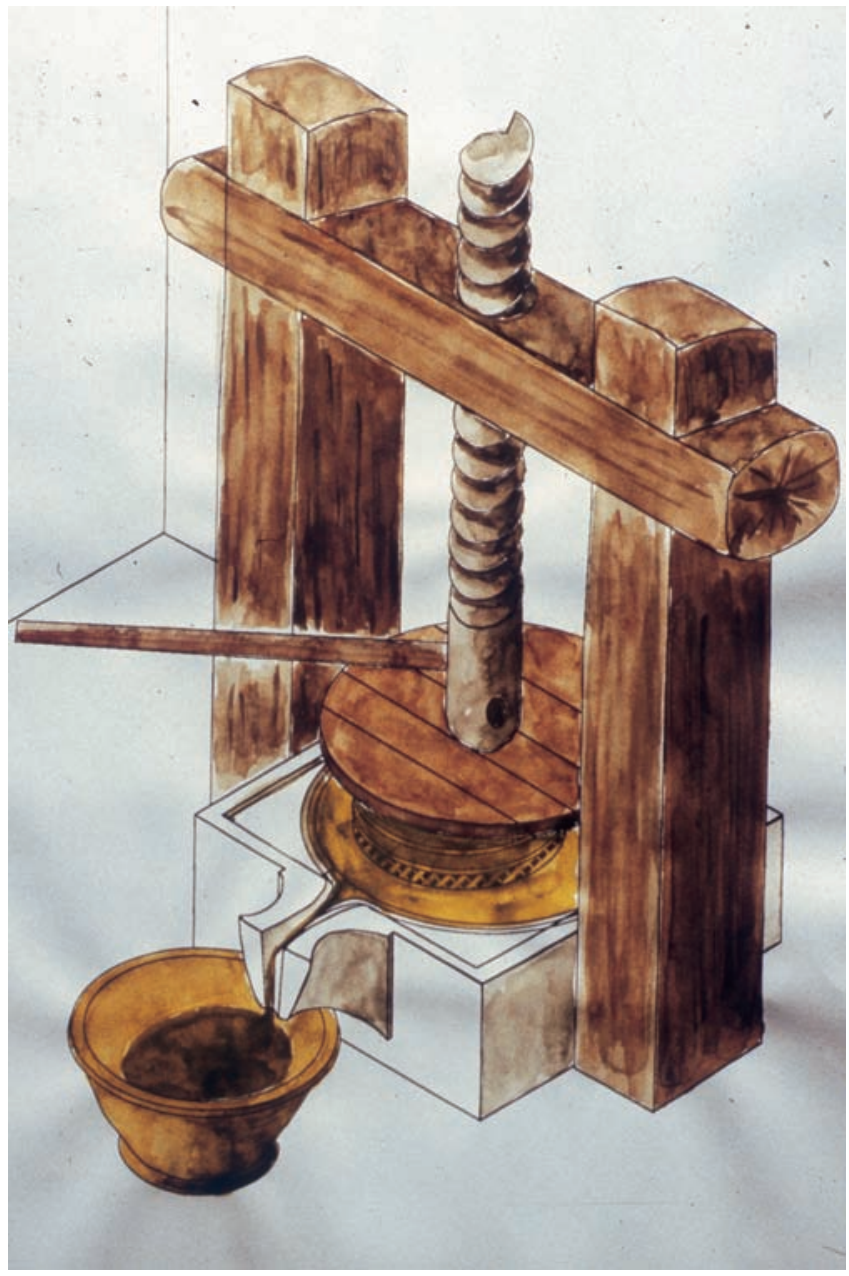

Fig. 16. Paestum : proposition de restitution d'un pressoir à vis (dessin J.-P. Brun) 


\section{Bibliographie}

Amouretti et alii 1984 : Amouretti (M.-C.), Comet (G.), Ney (C.), Paillet (J.-L.), À propos du pressoir à huile : de l'archéologie industrielle à l'histoire, MEFRA, 96, 1, 1984, p. 379-421.

Andreau 1973 : Andreau (J.), Histoire des séismes et histoire économique. Le tremblement de terre de Pompéi (62 apr. J.-C.), Annales E. S. C., 28, mars-avril 1973, p. 369-395.

Borgard et alii 2002 : BORGARD (PH.), BRUN (J.-P.), LeguillouX (M.), Tuffreau-Libre (M.), Activités archéologiques de l'École française de Rome 2001, Pompéi, Recherches sur les productions artisanales, MEFRA, 114, 2002, p. 470-481.

Brun 2003 : BRUN (J.-P.), Lartigianato dei profumi, in : Borgard (Ph.), Brun (J.-P.), Leguilloux (M.), TuffreauLibre (M.), Le produzioni artigianali a Pompei, Ricerche condotte dal Centre Jean Bérard, Rivista di Studi Pompeiani, XIV, 2003, p. 9-29.

Camodeca 1979 : Camodeca (G.), La gens Annia Puteolana in età giulio-claudia : potere politico e interessi comerciali, Puteoli III, 1979, p. 17-34.

Carra de Vaux 1988 : CARRA DE VAuX (B.) (éd.), Héron d'Alexandrie. Les mécaniques ou l'élévateur des corps lourds. Texte arabe de Qusta Ibn Luqa établi et traduit par B. Carra de Vaux, réimpression anastatique de l'édition de 1894, introduite par D.R. Hill, commentée par A.G. Drachmann, "Collection sciences et philosophie arabe», Paris, Les Belles Lettres, 1988.

Corallini 2001 : CoRALlinI (A.), Hercules domesticus, Immagini di Ercole nelle case della regione vesuviana (I. sec. A.C.-79 d.C.). Electa, Napoli (Studi della Soprintendenza archeologica di Pompei, 4), 2001.

De Caro 1979 : DE CARo (S.), Scavi nell'area fuori la Porta di Nola a Pompei, Chronache Pompeiane, 5, 1979 , p. 61.

Fiorelli 1860-1864 : Fiorelli (G.), Pompeianarum antiquitatum historia. Napoli 1860-1864 (3 vol).

Fiorelli 1875 : Fiorelli (G.), Descrizione di Pompei. Napoli, 1875.

Frederiksen 1959 : FREDERIKSEN (M.W.), Republican Capua, A social and economic study, PBSR, 27, 1959, p. $80-130$.

Fröhlich 1991 : FRÖHLICH (Th.), Lararien- und fassadenbilder in den Vesusvstädten. Untersuchungen zur volkstümlichen' pompejanischen Malerei, MDAIR Ergäzungsheft, 32, Mainz, Ph. von Zabern, 1991.

Greco 1986 : Greco (E.) (ed.), Aurigemma (S.), Spinazzola (A.), Maiuri (A.), I primi scavi di Paestum. Salerno, 1986.
Giordano \& Casale 1992 : GioRdano (C.), CASALE (A.), Profumi e acconciature in Pompei antica. Roma, Bardi, 1992.

Jashemski 1979, 1993 : JASHEMSKI (W. F.), The gardens of Pompeii, Herculaneum and Villas destroyed by Vesuvius. New Rochelle, Caratzas, t. 1, 1979 (372 p., 526 ill.) ; t. 2.1993 (432 p., 500 ill.).

Maiuri 1928-1929 : MAIURI (A.), Lavori di restauro della Soprintenza alle Antichità della Campania nel biennio 1927-1928, Bolletino d'Arte, VIII, 1928-1929, p. 512526.

Maiuri 1931 : MAIURI (A.), Herculaneum. Four years of discovery. Social life in Roman Italy, The Times, 6 novembre 1931, p. 15.

Maiuri 1932 : MAIURI (A.), Herculanum, Alpina, Paris, 1932. (Traduction française de Ercolano, "Visioni italiche», Istituto Geografico De Agostini, Roma-Novara, 1932).

Maiuri 1936 : MAIURI (A.), Herculaneum, «Itinéraires des musées et monuments de l'Italie», 53, Roma, Libreria dello Stato, 1936 (Anno XV E.F.).

Maiuri 1958 : MAIURI (A.), Ercolano. I nuovi scavi. (19271958), vol. 1, Roma, Libreria della Stato, 1958.

Mattingly 1990 : MATtingly (D.J.), Paintings, presses and perfume production at Pompeii, OJA, 9, 1, 1990, p. 71-90.

Pappalardo 1987 : PAPPALARDo (U.), La "Villa Imperiale" a Pompei, rapporto preliminare, DialArch. 5, 1987, p. $125-134$.

PPM : Pompei. Pitture e mosaici, Roma, Istituto per l'Enciclopedia Italiana, 1990-2003.

Scatozza Höricht et alii 1993 : SCATOZZA HÖRICHT (L. A.), Chianese (L.), Piccioli (C.), Sacchi (R.), Prime osservazioni ed analisi sul contenuto di alcuni recipienti in vetro rinvenuti nell'area archeologica di Pompei, in : Franchi dell'Orto (L.), Ercolano 1738-1988. 250 anni di ricerca archeologica. Roma, L'Erma di Bretchsneider, 1993, p. 551-564.

Spinazzola 1953 : Spinazzola (V.), Pompei alla luce dei nuovi scavi di via dell'Abbondanza, Roma, Libreria della Stato, 1953.

Tran Tam Tinh 1988 : Tran TAM Tinh (V.), La Casa dei Cervi à Herculanum. Roma, G. Breitschneider (Archeologica 74), 1988.

Villeneuve 1988 : Villeneuve (F.), Fouilles à Khirbet Edh-Dharih (Jordanie), 1984-1987 : un village, son sanctuaire et sa nécropole aux époques nabatéennes et romaines ( ${ }^{\text {er }}-\mathrm{IV}^{\mathrm{e}}$ siècles ap. J.-C.), CRAI, 1988, 2, p. $458-479$. 


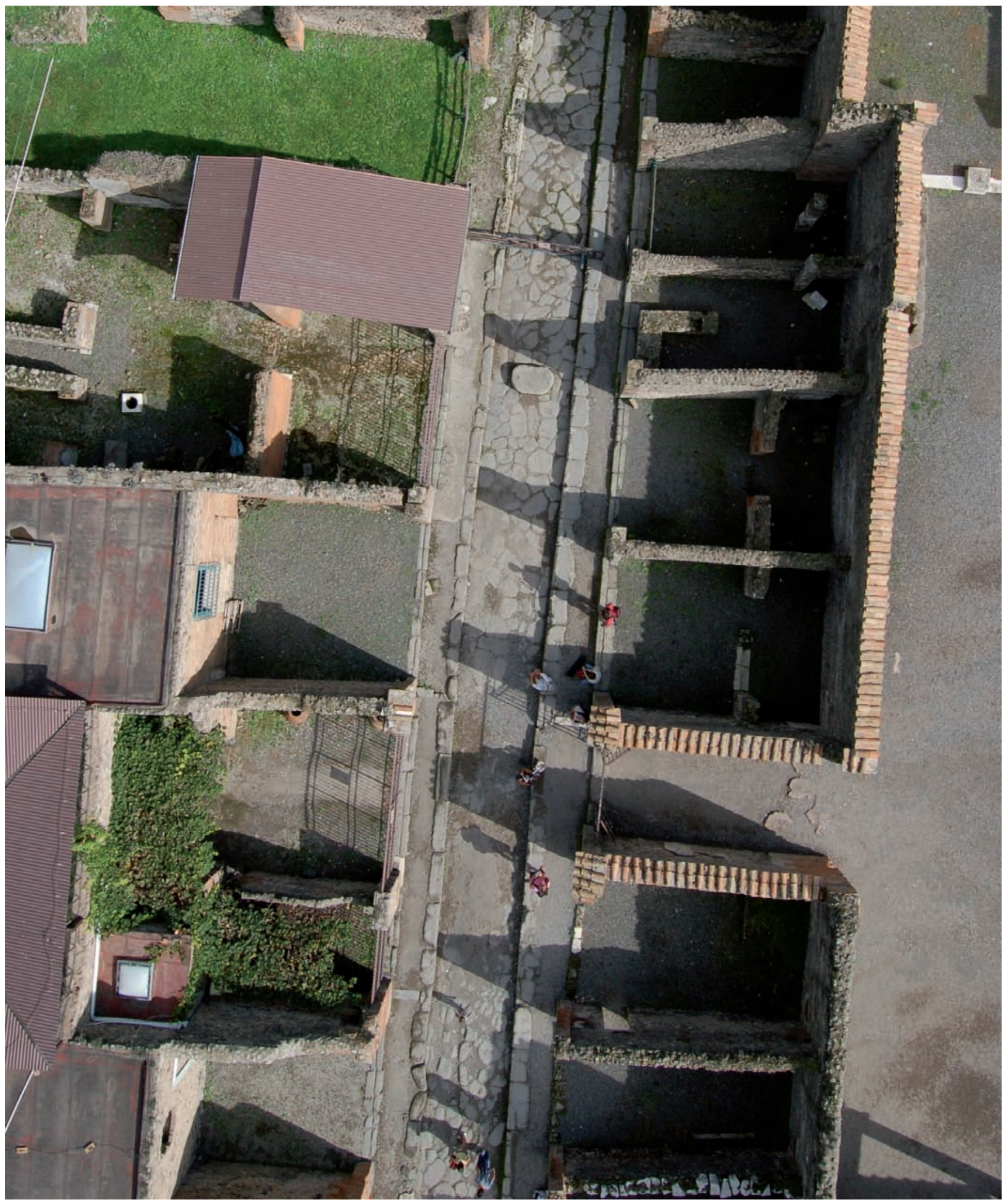

Fig. 17. La via degli Augustali à Pompéi : Rue des parfumeurs ?

La parfumerie fouillée en VII 4, 24-25 est située sous la toiture (cliché J.-P. Brun) 